\title{
Development of a new version of the Liverpool Malaria Model. II. Calibration and validation for West Africa
}

\author{
Volker Ermert ${ }^{1 *}$, Andreas H Fink', Anne E Jones ${ }^{2}$, Andrew P Morse ${ }^{2}$
}

\begin{abstract}
Background: In the first part of this study, an extensive literature survey led to the construction of a new version of the Liverpool Malaria Model (LMM). A new set of parameter settings was provided and a new development of the mathematical formulation of important processes related to the vector population was performed within the LMM. In this part of the study, so far undetermined model parameters are calibrated through the use of data from field studies. The latter are also used to validate the new LMM version, which is furthermore compared against the original LMM version.
\end{abstract}

Methods: For the calibration and validation of the LMM, numerous entomological and parasitological field observations were gathered for West Africa. Continuous and quality-controlled temperature and precipitation time series were constructed using intermittent raw data from 34 weather stations across West Africa. The meteorological time series served as the LMM data input. The skill of LMM simulations was tested for 830 different sets of parameter settings of the undetermined LMM parameters. The model version with the highest skill score in terms of entomological malaria variables was taken as the final setting of the new LMM version.

Results: Validation of the new LMM version in West Africa revealed that the simulations compare well with entomological field observations. The new version reproduces realistic transmission rates and simulated malaria seasons are comparable to field observations. Overall the new model version performs much better than the original model. The new model version enables the detection of the epidemic malaria potential at fringes of endemic areas and, more importantly, it is now applicable to the vast area of malaria endemicity in the humid African tropics.

Conclusions: A review of entomological and parasitological data from West Africa enabled the construction of a new LMM version. This model version represents a significant step forward in the modelling of a weather-driven malaria transmission cycle. The LMM is now more suitable for the use in malaria early warning systems as well as for malaria projections based on climate change scenarios, both in epidemic and endemic malaria areas.

\section{Background}

The World Health Organization (WHO) estimated that about two billion people, that is more than $40 \%$ of the total world population, are exposed to malaria [1]. Estimates in terms of 2009 revealed that this mosquitoborne disease causes about 225 million cases and 781,000 deaths annually. At least $90 \%$ of the worldwide malaria deaths occur in sub-Saharan Africa [2].

\footnotetext{
* Correspondence: vermert@meteo.uni-koeln.de

${ }^{1}$ Institute of Geophysics and Meteorology, University of Cologne, Cologne, Germany

Full list of author information is available at the end of the article
}

Malaria is a climate-sensitive tropical disease and hence climate exerts a strong impact upon the distribution of malaria transmission in space and time [3]. An assessment of current and future malaria risk is an important topic in the area of research relating climate to disease risk [4]. Reliable forecasts of epidemic malaria outbreaks on seasonal timescales [5] and assessments of disease vulnerability over decadal timescales are needed [6]. However, this requires the production of a weathermalaria modelling system. World-leading numerical weather forecast centres have already demonstrated useful skill in forecasts far beyond a month lead-time for 
some tropical regions [7], and climate projections are becoming more reliable [8]. Advances have also been achieved in weather- or climate-driven malaria modelling. For example, Hoshen and Morse [9] introduced the Liverpool Malaria Model (LMM), which is a mathematical-biological model of malaria parasite dynamics driven by daily temperature and precipitation data. However, further progress must be obtained in order to enable skilful malaria simulations for epidemic and endemic malaria regions based on meteorological data. For this reason, the present study introduces the LMM version of 2010 (henceforth called $\mathrm{LMM}_{2010}$ ), which simulates a more realistic spread of malaria in space and time and is hence a useful tool for a weather- or climate-disease modelling system.

In the first part of this study [10], an extensive literature review enabled the construction of a set of refined parameter settings (see Table 1) and an extended mathematical formulation of the LMM. Important sub-modules of the original LMM version were reviewed and updated. The oviposition, as well as the survival of immature mosquitoes, were adjusted to field conditions via the application of a fuzzy distribution model. In the second part of this study (this paper) previously undetermined model parameters are calibrated and the $\mathrm{LMM}_{2010}$ is validated by means of entomological and parasitological field observations from West Africa.

\section{Methods \\ Data sources \\ Time series of meteorological stations}

In the present study, temperature and precipitation measurements from synoptic weather stations across West Africa (see Figure 1) were used as LMM data input. Weather station data were gathered from the archive of the German Weather Service (DWD; German Weather Service) as well as from the Federal climate complex Global Surface Summary of Day version 7 (GSOD; from the US National Climate Data Centre) data. The meteorological time series were qualitychecked and missing values were filled according to a specific procedure, which is described by Ermert [11].

Table 1 LMM parameters and mathematical formulations

\begin{tabular}{|c|c|c|c|}
\hline sym & parameter & $\mathrm{val}_{2004}$ & $\mathrm{val}_{2010}$ \\
\hline$\overline{D_{g H}}$ & humid degree days of the gonotrophic cycle & 37.1 degree days & 37.1 degree days \\
\hline$D_{g L}$ & dry degree days of the gonotrophic cycle & 65.4 degree days & 65.4 degree days \\
\hline$T_{g H}$ & humid gonotrophic temperature threshold & $7.7^{\circ} \mathrm{C}$ & $7.7^{\circ} \mathrm{C}$ \\
\hline$T_{g L}$ & dry gonotrophic temperature threshold & $4.5^{\circ} \mathrm{C}$ & $4.5^{\circ} \mathrm{C}$ \\
\hline$R_{-}$ & 10-day accumulated precipitation threshold & $10 \mathrm{~mm}$ & $10 \mathrm{~mm}$ \\
\hline R. & rainfall laying multiplier & 1.0 & $\mathrm{NU}$ \\
\hline$\# E_{p}$ & number of produced eggs per female mosquito & $\mathrm{NU}$ & 120 eggs \\
\hline$\# E_{0}$ & number of oviposited eggs per female mosquito & NU & Eq. Two in [10] \\
\hline$U_{1}$ & lower threshold of unsuitable rainfall conditions (fuzzy distribution model) & NU & $0 \mathrm{~mm}$ \\
\hline$S$ & most suitable rainfall condition (fuzzy distribution model) & NU & $10 \mathrm{~mm}$ \\
\hline$U_{2}$ & upper threshold of unsuitable rainfall conditions (fuzzy distribution model) & NU & $500 \mathrm{~mm}$ \\
\hline CAP & cap on the number of fertile mosquitoes & 10,000 mosquitoes & 400 mosquitoes \\
\hline MMA & mosquito mature age & 15 days & 12 days \\
\hline$\eta_{d, \neg R}$ & rainfall independent immature daily mosquito survival probability & $\mathrm{NU}$ & $82.5 \%$ \\
\hline$\eta_{d}$ & daily immature mosquito survival probability (in \%) & Eq. Three in [10] & Eq. Four in [10] \\
\hline$p_{d}$ & daily mosquito survival probability (in \%) & Martens I (see [10]) & Martens II (see [10]) \\
\hline$p_{d \downarrow}$ & dry season mosquito survival probability shift & $\mathrm{NU}$ & $-10 \%$ \\
\hline$D_{s}$ & degree-days of the sporogonic cycle & 111 degree days & 111 degree days \\
\hline$T_{s}$ & sporogonic temperature threshold & $18^{\circ} \mathrm{C}$ & $16^{\circ} \mathrm{C}$ \\
\hline$a$ & human blood index & $50 \%$ & $80 \%$ \\
\hline$b$ & mosquito-to-human transmission efficiency & $50 \%$ & $30 \%$ \\
\hline$c_{a \rightarrow c}$ & adult-child conversion rate & $\mathrm{NU}$ & 0.5 \\
\hline HIA & human infectious age & 14 days & 20 days \\
\hline$r$ & daily human recovery rate & 0.0284 day $^{1}$ & $\mathbf{0 . 0 0 5 0}$ day $^{-1}$ \\
\hline GF & fraction of gametocyte carriers & NU & $50 \%$ \\
\hline c & human-to-mosquito transmission efficiency & $50 \%$ & $20 \%$ \\
\hline$t r_{i m}$ & trickle of the number of added infectious mosquitoes & 1.01 mosquitoes & 1.01 mosquitoes \\
\hline
\end{tabular}

LMM model parameters and mathematical formulations with regard to their original (Hoshen and Morse 2004) and new settings. Columns: sym: symbol of the model parameter; parameter: name of the parameter; unit: unit; val 2004 : $\mathrm{LMM}_{2004}$ value; val 2010 : $\mathrm{LMM}_{2010}$ value. Abbreviations: NU: not used. Parameter values in italics refer to calibrated values and those values and mathematical formulations in bold were determined in the first part of this study [10]. 
The resulting analysis provides continuous and qualitycontrolled temperature $(T)$ and rainfall $(R)$ time series between 1973 and 2006 for 34 synoptic weather stations in West Africa (see Figure 1 and Additional file 1).

Altogether 830 different sets of parameter settings of the LMM were forced by temperature and rainfall conditions derived from intermittent time series from 34 weather stations. The model was, therefore, subject to different climate conditions (cf. the varying temperature and rainfall values in Figure 2). The climates covered by the meteorological data set range from arid hot desert to tropical monsoon climates and therefore lead to various observed transmission levels of epidemic and endemic malaria. Fairly dry conditions in the Sahel, for example, resulted in the field in no malaria transmission at Diomandou Dieri (Senegal; $16^{\circ} 31^{\prime} N$; $14^{\circ} 39^{\prime} \mathrm{W}$ ) [12]. In contrast, nearly continuous rainfall caused year-round transmission in Cameroon at Etoa $\left(3^{\circ} 46^{\prime} \mathrm{N} ; 11^{\circ} 29^{\prime} \mathrm{E}\right)$ [13]. The temperature range of this meteorological data set lie, for most stations, well above $20^{\circ} \mathrm{C}$ (cf. Figure 2) inhibiting the model validation for the lower malaria temperature limit of about $16^{\circ} \mathrm{C}$ [14].

\section{Regional climate simulations}

Driving the LMM with gridded data is needed for the validation of the simulated malaria spread in Africa. Due to the lack of gridded daily temperature and rainfall data from ground observations, data from a so-called regional climate model are used. Such a model is able to more accurately represent spatial variations of the atmosphere than so-called global atmosphere-ocean general circulation models.

Gridded LMM simulations for Africa were based on meteorological data from three ensemble runs between 1960 and 2000 from the REgional MOdel (REMO) [15]. REMO is a hydrostatic, limited area model using primitive equations, which are solved on 20 hybrid atmospheric levels [16]. At the lateral and lower boundaries REMO was nested into ECHAM5/MPI-OM (European Centre HAmburg Model, 5th generation/Max-PlanckInstitute-Ocean Model) global coupled climate model simulations that were forced with the observed greenhouse gas increase [17-19]. The space-time resolution of REMO data used in the present study was $0.5^{\circ}$ and daily values, respectively.

Expected systematic model errors in annual precipitation and in simulated temperatures were considerably reduced using bias correction, ensuring realistic data input for the malaria simulations [11]. The bias-corrected data are later used to drive gridded malaria runs in order to compare the original and new LMM versions.

\section{Entomological and parasitological observations}

In terms of malaria modelling, the entomological and parasitological data are of particular interest since

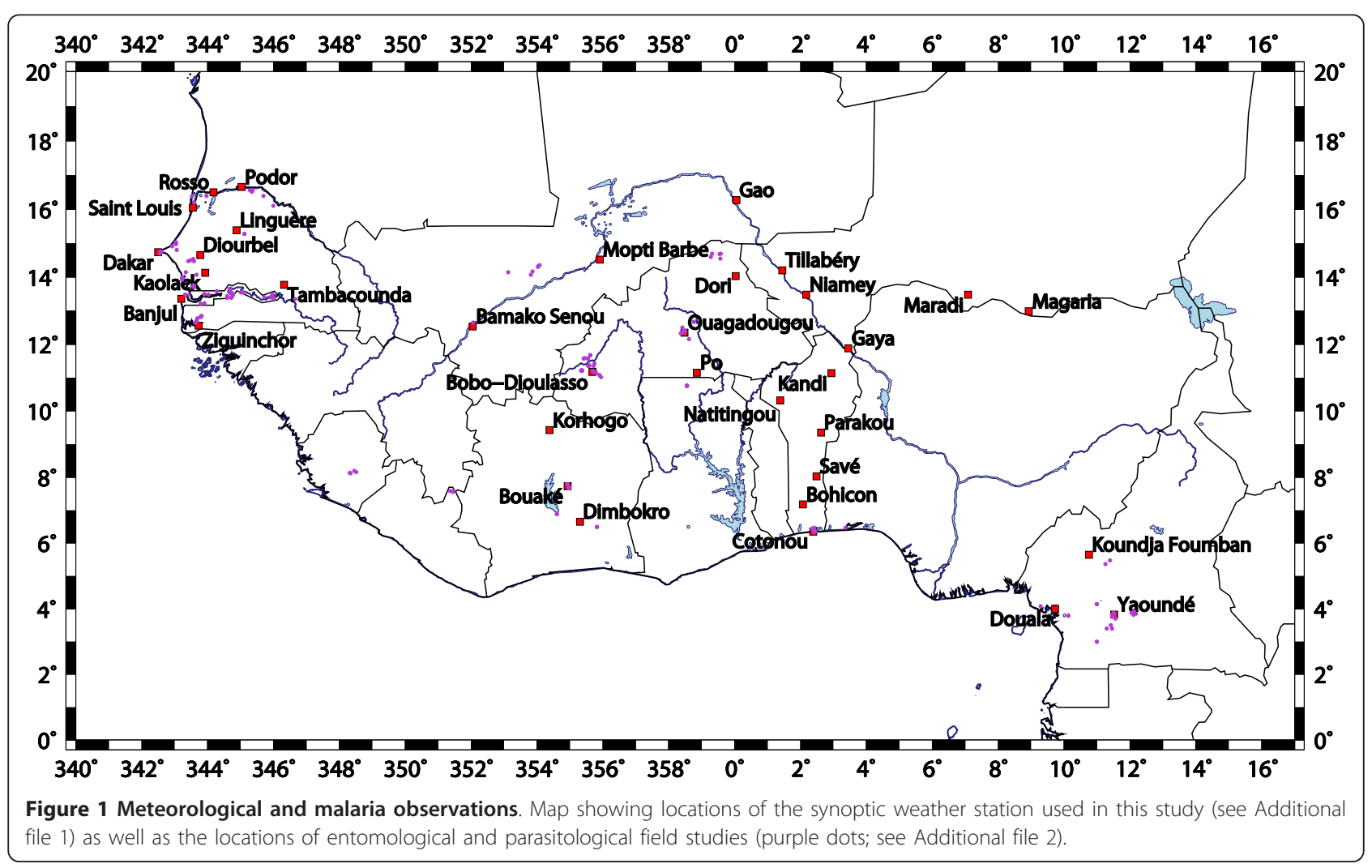




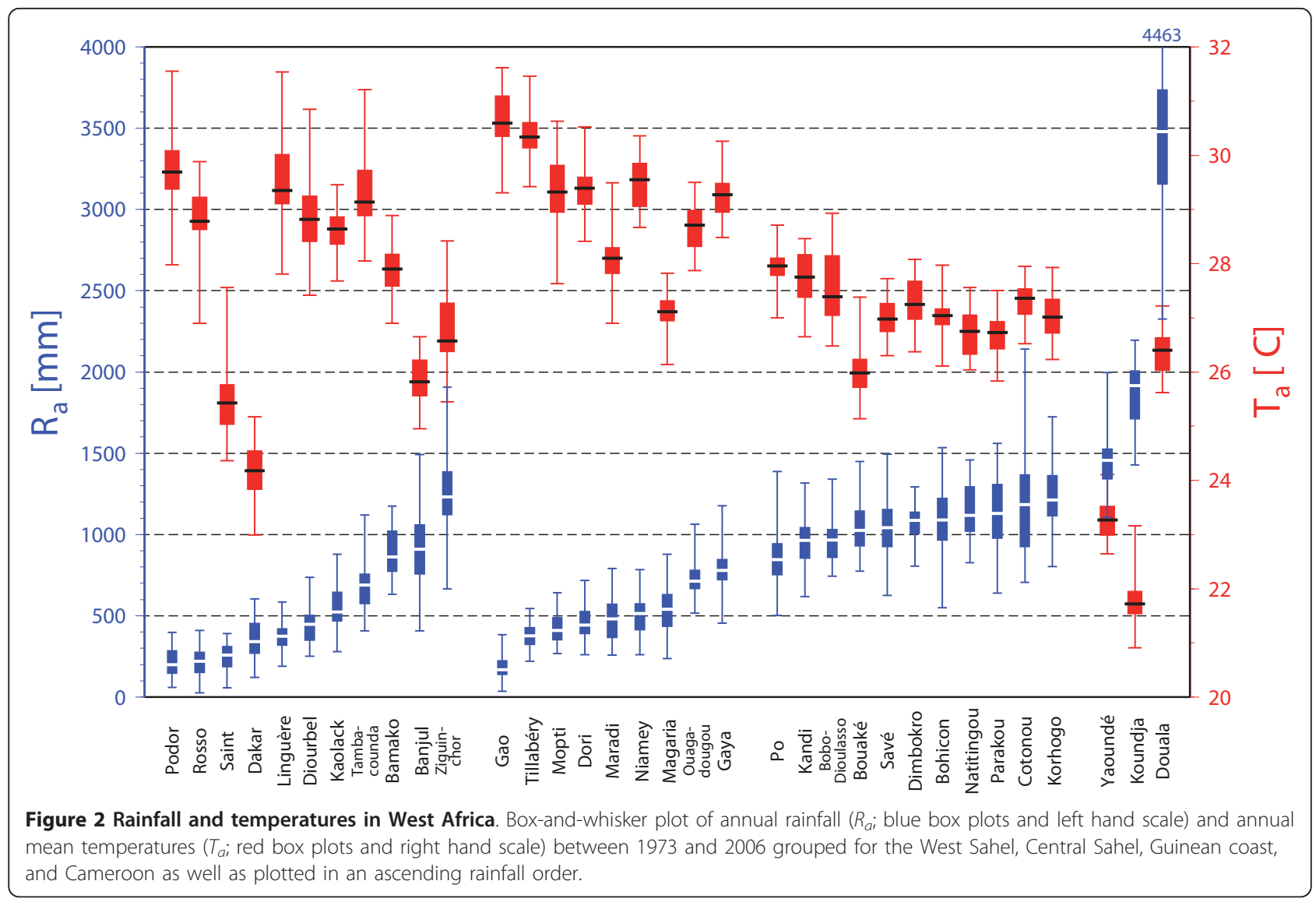

malaria models have to undergo validation procedures. The present study was restricted to West Africa since this area was one of the focus regions of the IMPETUS project (Integrated Approach to the Efficient Management of Scarce Water Resources in West Africa) [20]. Numerous published malaria observations were extracted from the literature.

It was shown that population density is an important factor of malaria transmission $[21,22]$. In addition, crop irrigation strongly impacts malaria and its seasonality [23-26]. Also malaria and vector control such as the usage of impregnated bed nets are able to reduce transmission [27-31]. In the present study, field observations were only included for which a rural environment can be assumed from the information given in the publication (see Additional file 2 which includes also data from non-rural sites). Urban and irrigated areas and those when permanent streams influenced transmission were excluded (except for the locations in Cameroon, where this is nearly always the case). The few sites subject to vector control or other local or regional interventions were not used. This procedure ensured that other factors than climate, which influence malaria transmission, were excluded to the extent possible.
Entomological malaria field studies frequently sample biting mosquitoes on humans [32]. Standard measures include the so-called Human Biting Rate (HBR), which is the number of mosquito bites per human per time. However, only female mosquitoes with sporozoites in their salivary glands are able to infect humans. This fraction of the biting females is called CircumSporozoite Protein Rate (CSPR). Multiplying HBR with CSPR results in the so-called Entomological Inoculation Rate (EIR), which is defined as the number of infectious mosquito bites per human per time [33]. Only months revealing infectious mosquito bites ( $E I R$ values above zero) are usually used to define the malaria season at a certain location [34]. By contrast, parasitological malaria studies usually measure the asexual parasite ratio $(P R)$ representing the proportion of the survey population, which is positive for the malaria parasite [35]. The literature was, therefore, reviewed in terms of malaria field studies (see Additional file 2).

Wherever applicable, observations were taken into account with regard to eleven different entomological and parasitological variables (these are: the annual Human Biting Rate $\left(H B R_{a}\right)$, the annual Entomological Inoculation Rate $\left(E I R_{a}\right)$, the annual mean CircumSporozoite Protein 
Rate $\left(C S P R_{a}\right)$, the length, start, and end of the malaria season (Seas, SSeas, and ESeas, respectively), the length of the main malaria season (MSeas; i.e. the number of months in which $75 \%$ of $E I R_{a}$ is recorded [36]), the month of maximum transmission (XSeas; i.e. the month with the largest entomological inoculation rate), the annual mean, maximum, and minimum of the asexual parasite ratio $\left(P R_{a}\right.$, $P R_{\text {max }}$ a and $P R_{\text {min }}$ a , respectively). Included are the literature references as well as some basic features such as the name, geographical position, land-use of the study site, and the time period. Note that all these malaria variables can be computed from the LMM output.

\section{Definition of malaria seasonality}

The definition of the malaria season is based on the monthly Entomological Inoculation Rate $\left(E I R_{m}\right)$, which is observed from field studies. The introduction of the malaria parasite into the LMM is assured by a constant influx of new infectious mosquitoes resulting in EIR values that might exceed those of low transmission areas. In order to compare 'truly' modelled EIR values with field observations, the artificial EIR values were removed via two separate LMM runs. The standard run results in a mixture of bites from the added infectious mosquitoes and those which were infected when they bit infectious humans in the simulation. The artificial $E I R$ values were produced in a second run when only the added infectious mosquitoes were biting in the model, achieved by setting the number of produced eggs per female mosquito $\left(\# E_{p}\right.$ ) to zero. Here, the oviposition of Anopheles females is prohibited and the mosquito population is therefore not able to grow. The subtraction of the EIR values of the second run from that of the standard run results finally in the elimination of artificial EIR values. Note that the same procedure was used with regard to the computation of the human biting rate $(H B R)$.

In field studies, the malaria season is mostly determined on a monthly scale and is usually referred to months with $E I R_{m}$ values above zero [34]. For this reason, in the model the malaria season starts (SSeas) by definition in the first month with an $E I R_{m}$ value of at least 0.01 infectious bites per human. According to the formulation of the model, the arbitrary EIR value of 0.01 means that during a 30-day month at least one out of the 100 modelled humans is bitten by an infectious mosquito. In West Africa, the malaria season usually ends when the number of mosquitoes decreases at the end of the rainy season. Therefore, mosquito biting is reduced to such low numbers that the EIR values are reduced to zero indicating that transmission has ceased. Consequently, the last month during the transmission period defines the end of the malaria season (ESeas). Some individual years also reveal year-round or even no malaria transmission for certain locations. The length of the malaria season (Seas) is therefore the number of months with $E I R_{m}$ reaching at least 0.01 infectious bites. For each site or grid box additionally the length of the main transmission season (MSeas) is defined as the number of months in which $75 \%$ of $E I R_{a}$ is transmitted, an index which was used by Hay et al. [37]. Where possible the month with the maximum malaria transmission (XSeas) is identified as the month with the highest $E I R_{m}$ value.

The use of the transmission threshold of 0.01 infectious mosquito bites per human per month ensures the attainment of a reasonable transmission level in the model that can be compared with observations from the literature. However, the definition of the malaria season in the model might not be directly comparable to field studies since observations are subject to a certain detection limit. That is due to the fact that field experiments do not continuously measure biting rates (at best two times in each week of the field campaign) and that these field studies do not account for every human of the population of the study site.

\section{Definition of the validation}

For the model validation, the LMM runs are driven by weather observations from meteorological stations in West Africa. The malaria model simulations are compared against observations from more than 200 sites using up to eleven different entomological and parasitological variables. The skill of the malaria simulations is measured by a problem-adapted skill score (see below). The performance of every particular set of parameter settings of the model is measured for each of the eleven malaria variables by this skill score $(S C(x))$. In addition, the performance of a set of parameter settings with regard to all malaria variables is quantified by the sum of the eleven skill scores $(S C(a l l))$. This approach enables the production of a ranking of different sets of parameter settings. Finally, the most suitable set of parameter settings in terms of the skill score determines the new LMM version.

The LMM was run with meteorological time series covering 1973 to 2006 discussed above. Therefore, each simulation at a particular station produced 34 annual values for every considered variable (e.g. $\left.E I R_{a}\right)$. Since malaria conditions are different between rural and urban areas [21], only data from rural field sites were used for the LMM validation. On the other hand, entomological data are never measured continuously because of the amount of work required in practice [38]. For this reason, no single long time series of malaria observations exist. However, field observations of a subset of the nine malaria variables were frequently found in the area of the considered weather stations. Unfortunately, 
such malaria data has not been detected for all the used stations. For example, in the vicinity of Parakou (Benin) no field observations were extracted from the literature.

A further data issue that must be taken into account arises from the fact that the malaria field measurements were not performed at the locations of the weather stations. However, observation sites are available in the vicinity of the weather stations meaning that they are located in same climatic zones (Figure 1). In this context, it must be noted that precipitation can differ greatly between locations just few kilometres apart, but meteorological stations are much more coarsely distributed $[39,40]$. Also different environmental conditions (e.g. state of land surface) complicate the comparison between simulations and field observations. Shaman and Day [39] allude to the mismatch between the scales at which disease vectors respond to hydrologic variability and the scales at which hydrologic variability is actually monitored. For all these reasons, a reproduction of the field values of particular years, for example, of transmission rates is not expected by the LMM. In addition, it implies that paired annual correlations at single stations are not useful. Therefore, the usage of standard skill scores, used to determine the skill in weather forecasting [41], is not possible with the data sets that are available. In addition, statistical moments of the model and observational malaria data cannot be statistically meaningful tested due to the low sample size of the field observations. In order to overcome these issues, a subjective problem-adapted skill score is defined in the following section.

\section{Definition of a skill score}

A special validation procedure must be defined. It must be considered that simulations will not be able to reproduce single field observations. However, it is possible that the LMM simulates about the correct mean and variability of malaria variables, as observed in the field, since the weather station and the assigned field sites encounter similar climate conditions. In the following, location names refer to the name of the weather station, even if malaria observations from a nearby field site are discussed. The assignment of the weather stations to the malaria study sites is given in Additional file 2.

The evaluation claims that the simulated LMM values are comparable to field observations in reproducing the malaria-climate relationship across a range of malaria variables for the locations investigated in this paper. The validation procedure controls if, in general, the values of the model simulation agree with the field observations. Also the quantity of available observations is taken into account. The following six criteria define a problemadapted scoring system (see also Table 2):

The evaluation of the model runs is performed by means of a skill score (SC), which considers the number of available observations for each of the eleven malaria variables. Stations with at least five observations contribute at maximum three or four points to the calculated skill score. Weather stations with fewer field measurements add one to three points to the skill score (cf. Table 2). No score is added when stations reveal no observations. The validation method also makes allowances for the uncertainty of the year-to-year variability.

Table 2 Criteria in terms of the evaluation of LMM simulations

\begin{tabular}{|c|c|c|c|c|c|}
\hline \# & name & description & $\mathrm{n}_{\mathrm{obs}}$ & points & var \\
\hline 1 & overlap & Any observation is included in the simulated range & $\geq 1$ & +1 & all variables \\
\hline 2 & enclosure & Every observation is included in the simulated range & $\geq 2$ & +1 & all variables \\
\hline 3 & $\begin{array}{l}\text { median } \\
\text { enclosure }\end{array}$ & The observed median is included in the simulated range & $\geq 3$ & +1 & $\begin{array}{l}H B R_{a} E I R_{a} C S P R_{a} \text { Seas } \\
\text { MSeas } P R_{a} P R_{\text {min, }} P R_{\text {max }, a}\end{array}$ \\
\hline 4 & $\begin{array}{l}\text { median } \\
\text { quartile } \\
\text { enclosure }\end{array}$ & $\begin{array}{l}\text { The observed median is located within the lower and upper quartile of the } \\
\text { simulations }\end{array}$ & $\geq 5$ & +1 & $\begin{array}{l}\text { HBRa EIRa CSPR } \text { Seas } \\
\text { MSeas } P R_{a} P R_{\text {min, }} P R_{\text {max }, a}\end{array}$ \\
\hline 5 & penalty & The simulations exceed the one and a half time maximum of all field observations & $\geq 1$ & -5 & $H B R_{a} E I R_{a}$ \\
\hline \multirow[t]{6}{*}{6} & frequency & $\begin{array}{l}\text { I: The observations as well as the simulations show the same month maximum } \\
\text { occurrence of the monthly entomological inoculation rate }\left(E I R_{m}\right) \text {. }\end{array}$ & $\geq 3$ & +1 & SSeas ESeas XSeas \\
\hline & & $\begin{array}{l}\text { II: The majority of the observations and simulations show no or yearround } \\
\text { transmission, respectively. }\end{array}$ & & & \\
\hline & & III: The observations and simulations reveal mostly no transmission. & & & \\
\hline & & $\begin{array}{l}\text { IV: The month showing the most field observations of multiple years of } E I R_{m} \text { is } \\
\text { identical to the particular month resulting from the simulation. }\end{array}$ & & & \\
\hline & & SSeas/ESeas: Criterion I or \|| & & & \\
\hline & & XSeas: Criterion III or IV & & & \\
\hline
\end{tabular}

Criteria in terms of the evaluation of LMM simulations which are based on field observations in the area of synoptic stations. The malaria runs are rated separately for each station. Every fulfilled criterion increases the score of such a model run by one point. The sum of the achieved points at all stations and from the eleven entomological and parasitological variables finally add to the skill score of a particular LMM set of parameter settings (SC(all)). Columns: \#: criteria number; name: short term; description: criterion description; $\mathrm{n}_{\text {obs: }}$ lowest number of available observations needed to fulfil the criterion; points: assigned number of points; var: malaria variables for which the particular criterion are applied. 
A proper analysis would only be possible for numerous available observations per station. In fact, an estimate of the frequency distribution of observed malaria parameters is not feasible from the few records available.

The computed skill score is based on the probability that observations, assigned to particular weather stations, fall into certain ranges of simulations (Table 2). It is expected that any observation is included in the range of the model simulations with regard to this station data (criterion 1: overlap). Of course, the model version performs better when every observation is enclosed in the simulated range (criterion 2: enclosure). Where there are at least three available records the observed median is also calculated. The confidence in the run increases when the observed median is contained in the range of the model simulations (criterion 3: median enclosure). The reliability of the median estimate increases for sites where five observations or more field measurements exist. Only for at least five available records the condition has to be met that the observed median falls within the range of the lower (25th percentile) and upper quartile (75th percentile) of the 34 simulated annual values (criterion 4: median quartile enclosure). For model integrations with different parameter settings, every fulfilled criterion at a weather station adds one point to the skill score of a particular malaria variable.

This rating system might favour model versions generating unrealistic high values and a strong interannual variation. This fact is countered by another criterion that eliminates unrealistically high entomological values (criterion 5: penalty). Five penalty points are applied to the skill score of $H B R_{a}$ and $E I R_{a}$, when any simulated value exceeds one and a half times the maximum of all available field measurements (see Table 2). This threshold seems to be a reasonable measure for the restriction of simulated values. Sets of parameter settings leading to unrealistic high biting rates $\left(H B R_{a}\right.$ and $E I R_{a}$ values) are rejected.

The third and fourth criteria are not calculable for three of the variables under certain circumstances (cf. Table 2): the start (SSeas) and end (ESeas) of the malaria season (for which the criteria are undefined in the cases of no transmission or year-round transmission), and the month of maximum transmission (XSeas) (for which they are undefined in the case of no transmission). For these variables, the maximum of the frequency distribution is compared instead (criterion 6: frequency). Regarding SSeas and ESeas, a model version is given an additional score of one point when both the observations as well as the simulations show the same month of maximum occurrence. A point is also gained when the majority of the observations and simulations show no or year-round transmission, respectively. For $X$ Seas, a model version can achieve one additional point for a weather station either if both the observations and simulations reveal mostly no transmission or if the month showing the most field observations of multiple years of maximum $E I R_{m}$ values is identical to the particular month resulting from the simulation (e.g. if for a single station the strongest transmission values are both mostly observed and simulated for August).

For each weather station, a skill score is determined for every set of parameter settings and for every one of the eleven entomological and parasitological variables (e.g. for Ouagadougou the skill score in terms of $E I R_{a}$, i.e. $S C\left(E I R_{a}\right)$, is 4 for the $\mathrm{LMM}_{2010}$; see Figure 3). The skill scores from the weather stations add up to the skill score of a particular variable $\left(S C(x)\right.$; e.g. $S C\left(E I R_{a}\right)$ is 41 for the $\mathrm{LMM}_{2010}$; see Figure 3). Finally, the sum of all skill scores of a particular set of parameter settings is calculated (i.e. $S C($ all $)$, which is 279 for the $\mathrm{LMM}_{2010}$ ).

For each particular station and each variable a certain number of points are achievable (e.g. 54 points can be reached in terms of $S C(E I R a)$ ). As stated above this optimum depends on the availability of field observations (see Table 2), which differs for each weather station and each malaria variable. The minimum number of possible points is zero for stations, with no available field observations (e.g. see Parakou in Figures 3, 4, and 5). At maximum four points can be achieved, when at least five observations are available. For example, numerous field observations are available for Bobo-Dioulasso (see Figure 3, 4, and 5). In case of SSeas, ESeas, and XSeas at maximum only three points can be achieved since only three criteria are applied for these variables (see Table 2).

\section{Results}

In the following, undefined parameters of the LMM are calibrated by means of West African field observations. A two step approach is used in order to reduce the degrees of freedom of the model within each step. The performance of the model runs is measured by the problem-adapted scoring system. At the end, the set of parameter settings revealing the highest skill score is selected. Finally, the simulations of the $\mathrm{LMM}_{2004}$ and $\mathrm{LMM}_{2010}$ are compared to field observations of the eleven entomological and parasitological malaria variables.

\section{Calibration of the LMM}

The majority of the model parameters were determined in the first part of this study [10]. However, some parameters were not allocatable due to a large spread in their published values or due to the lack of data. These undetermined values were two of the three parameters ( $S$ and $U_{2}$ ) of the fuzzy distribution model. Here, $S$ defines the most suitable 10-day accumulated rainfall conditions for mosquito breeding, meaning rainfall provides open water surfaces, which tend not to be flushed 


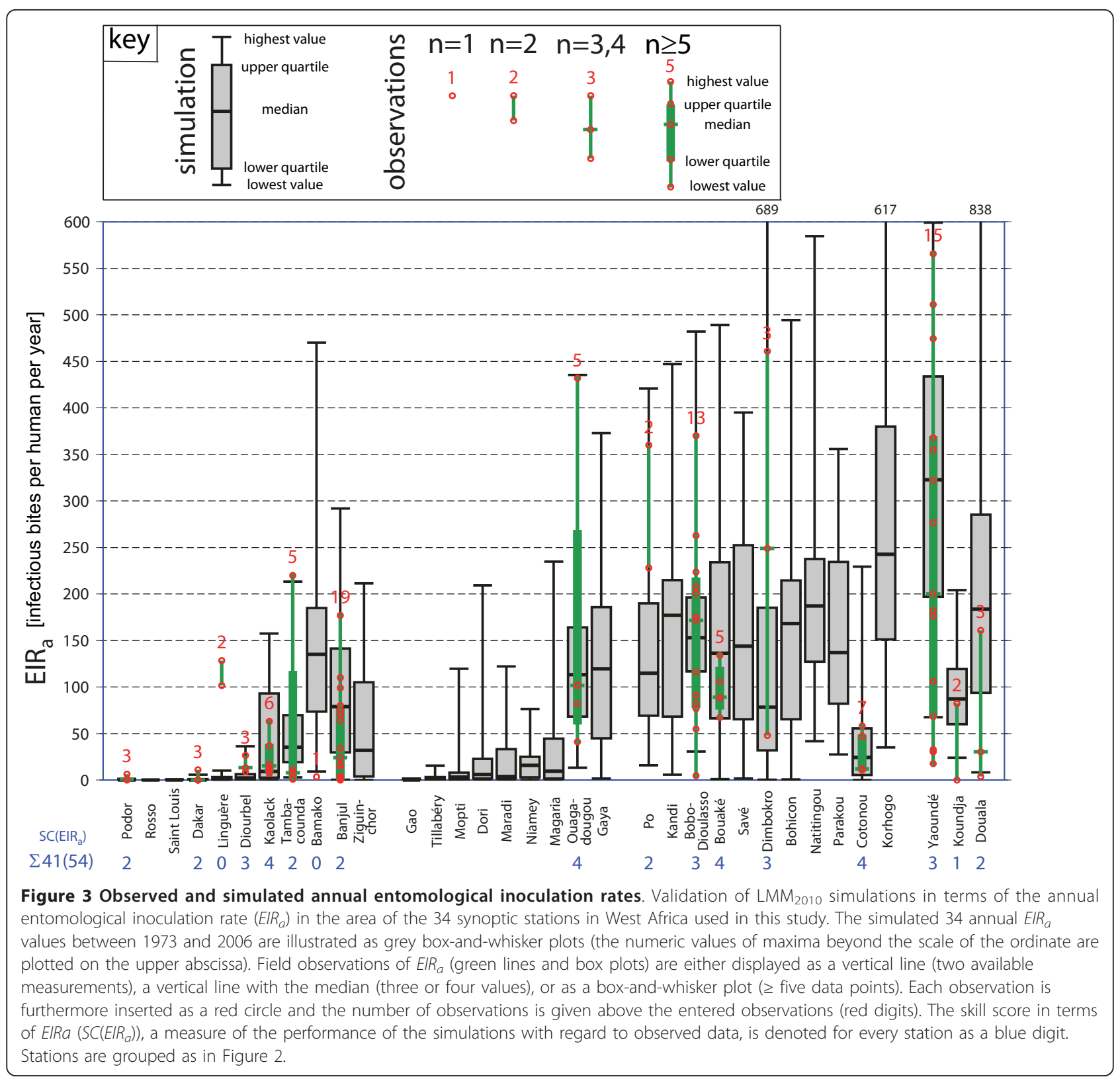

out by heavy rainfall. $U_{2}$ defines the upper limit of suitable rainfall conditions above which all breeding habitats are flushed out. Note that $U_{1}$, which is the lower limit of suitable rainfall conditions, was simply set to $0 \mathrm{~mm}$ since under prolonged dry conditions ephemeral water bodies will dry up [10].

In addition, the number of produced eggs per female mosquito $\left(\# E_{p}\right)$, the cap on the number of fertile mosquitoes $(C A P)$, as well as the shift off relative to the dry season mosquito survival probability $\left(p_{d \downarrow}\right)$ are undetermined. Here, $p_{d \downarrow}$ is used to reduce vector survival during dry atmospheric conditions [10]. In the first part of this study [10], some parameter values were estimated from the range of published data and few were based on educated guesses, this might have resulted in some false estimation of these predefined parameters. However, the following calibration of the remaining parameters will largely compensate such inaccurate assessments.

As outlined above, the LMM calibration was undertaken for weather conditions at meteorological stations and included two general steps: (i) The initial experiment enabled a rough estimate of realistic parameter values and allowed the setting of two undetermined parameters. (ii) The second set of model runs permitted a final adjustment of the remaining model parameter settings. In order to simplify the calibration procedure, 


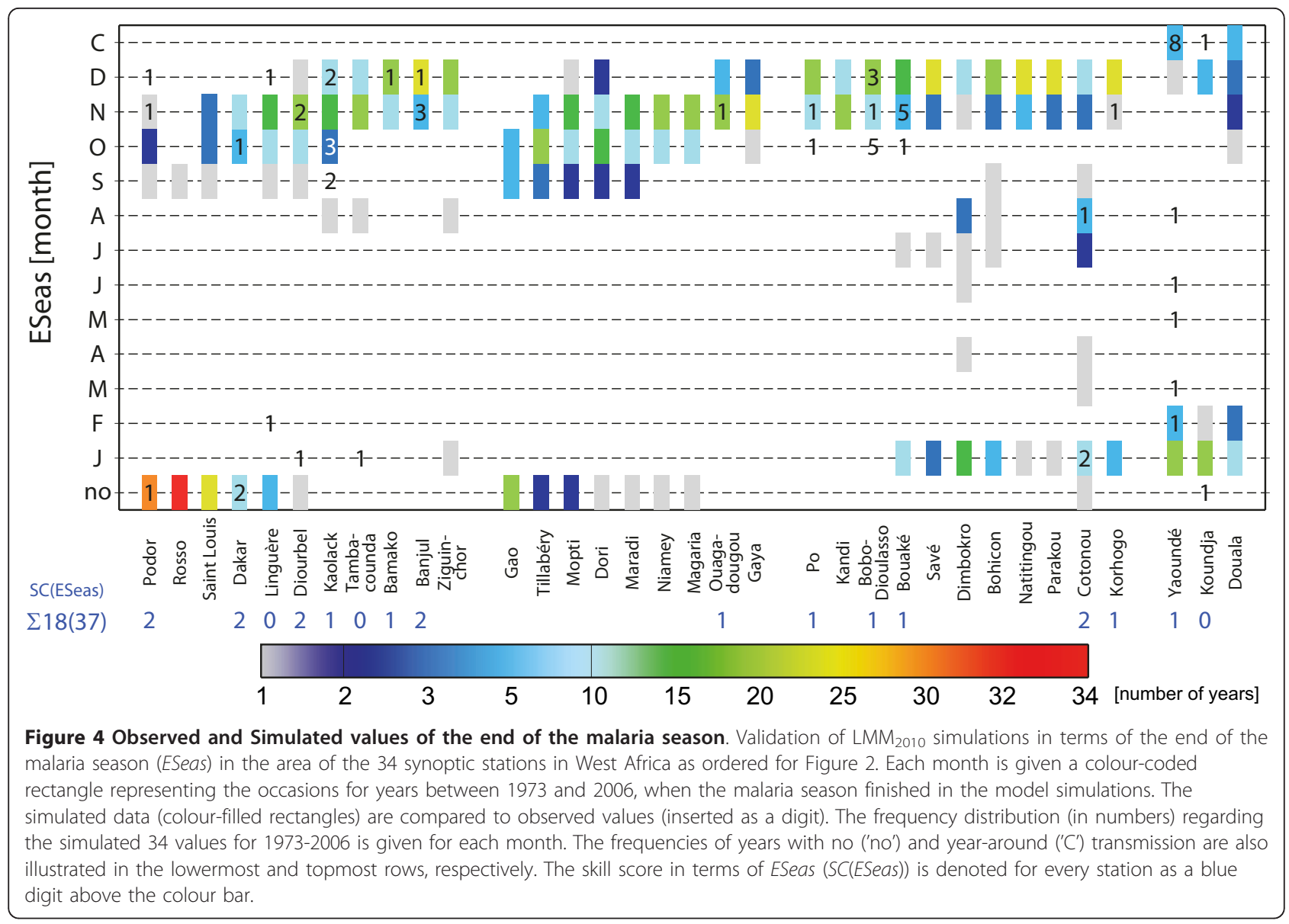

$p_{d \downarrow}$ was initially set to zero. Altogether three different settings were used for $U_{2}$ (i.e. the upper limit of suitable rainfall conditions in terms of larval breeding of the fuzzy distribution model). Ahumada et al. [42] defined extreme rainfall as more than $255 \mathrm{~mm}$ of cumulative rainfall throughout a period of three days. Their model markedly reduces the mosquito population under excessive rainfall. According to a 10-day period, which is used by the fuzzy distribution model, this suggests a $U_{2}$ value of about $500-1000 \mathrm{~mm}$. In fact, $U_{2}$ was either set to 500,750 , or $1000 \mathrm{~mm}$.

\section{First step}

For the isolation of particular sets of parameter settings the remaining parameters $\left(S, U_{2}, \# E_{p}\right.$, and $\left.C A P\right)$ were simultaneously varied $\left(S: 5,10,15,20\right.$, and $30 \mathrm{~mm} ; U_{2}$ : 500, 750, and $1000 \mathrm{~mm}$; $\# E_{p}: 50,75,100,125$, and 150 eggs; CAP: 250, 500, 750, 1000, and 2000 fertile females). The various LMM simulation runs were compared to data from the eleven entomological and parasitological variables (see Additional file 2), these are: $H B R_{a}, E I R_{a}, C S P R a$, SSeas, ESeas, Seas, $P R_{a}, P R_{\text {max a a }}$, and $P R_{\min }, a$. The first 375 (originating from the combination of $5 \cdot 3.5 .5$ settings) different LMM settings were ranked with regard to the skill score of all variables $(S C$ $(a l l))$ and in terms of the two entomological variables $H B R a$ and $E I R a$ (i.e. $S C\left(H B R_{a}, E I R_{a}\right)=S C\left(H B R_{a}\right)+S C$ $\left.\left(E I R_{a}\right)\right)$. Here, $S C\left(H B R_{a}, E I R_{a}\right)$ is measuring the skill of the model simulation in terms of both $H B R_{a}$ and $E I R_{a}$.

The ranking of sets of parameter settings with regard to $S C\left(H B R_{a}, E I R_{a}\right)$ showed that $S$ (i.e. the most suitable rainfall condition in terms of larval breeding of the fuzzy distribution model) affects mainly the spread of malaria in the northern part of the Sahel, for example, at various stations in Senegal [11]. In these dry areas the growth opportunity of the mosquito population is strongly suppressed by the fuzzy distribution model when $S$ is set to high values. In this case, fewer suitable breeding sites are assumed in the model reducing the number of eggs entering and of larvae surviving the aquatic stages [10]. Obviously, $S$ has to be set to relatively low values in order to keep malaria going in the northern Sahelian zone. However, too low $S$ values seem to be unrealistic since the potential evaporation in tropical Africa usually exceeds several millimetres per day. The optimal 10-day rainfall of $S$ is finally fixed to $10 \mathrm{~mm}$ since this value still enables the 


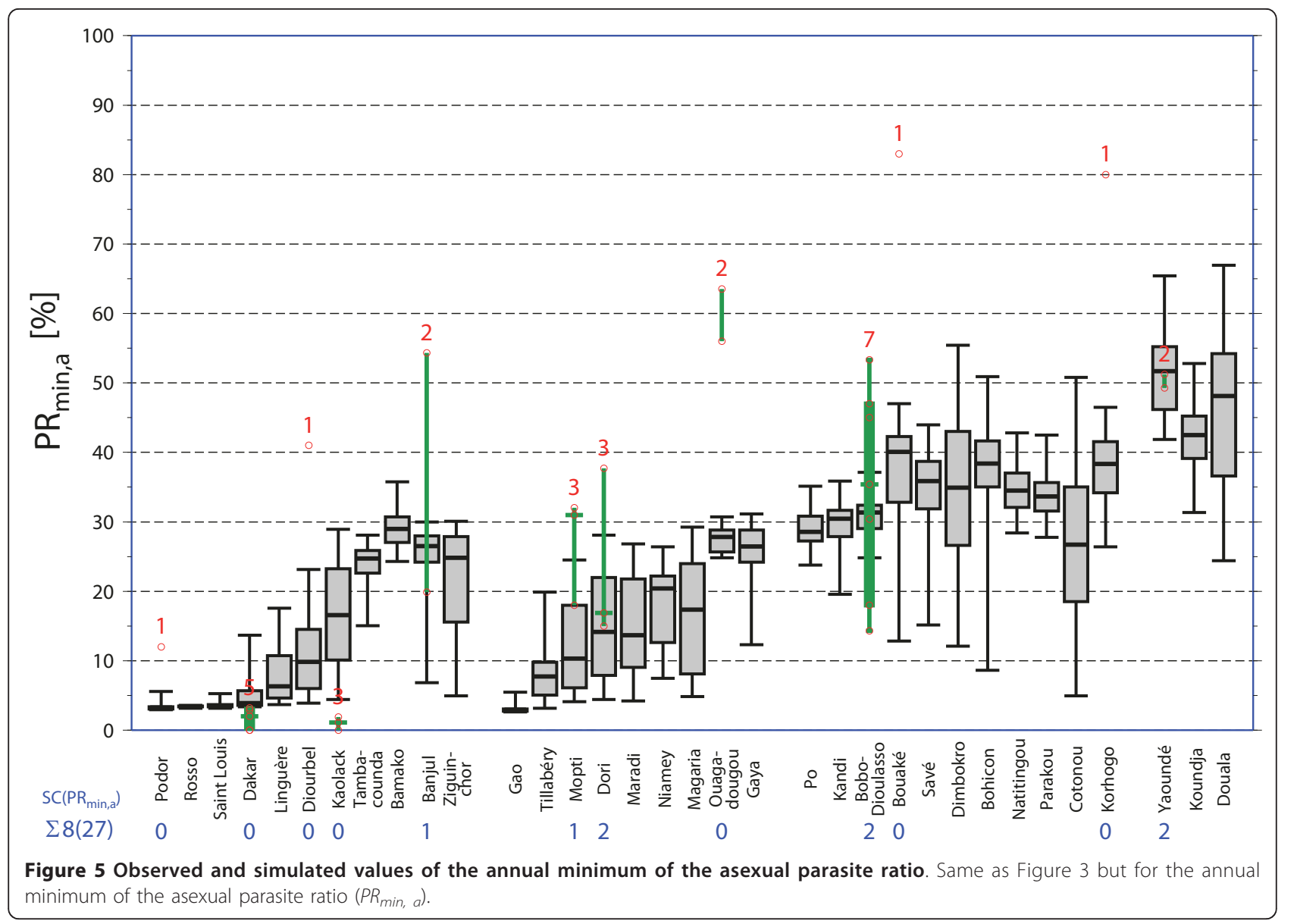

simulation of malaria in the northern part of the Sahel (see step 1.1 in Table 3). This analysis shows the clear need for the validation of the model under different rainfall conditions.

The evaluation of the performance of the 375 model versions also enabled a final setting of $U_{2}$ since highest skill scores were exclusively generated by a value of $500 \mathrm{~mm}$ [11]. The lowest skill scores were attained by settings with high values of $C A P, \# E_{p}$, and $U_{2}$, as well as low values of $S$, which apparently evoke large mosquito populations (see Additional file 3 and step 1.2 in Table 3).

A closer analysis of the data revealed that malaria transmission rates in the Sahel are fairly sensitive to the setting of $\# E_{p}$ (i.e. the number of produced eggs per female mosquito) [11]. The median $H B R a$ value rises, for example, at Linguère, Mopti, and Diourbel from less than 100 to several thousand bites per year, when $\# E_{p}$ increases from 50 to 150 eggs. Unfortunately, only nine field observations of $H R B_{a}$ and $E I R a$ are available north

Table 3 Overview of calibration experiments

\begin{tabular}{|c|c|c|c|c|}
\hline step & Parameter & area & SC & result \\
\hline step 1 & $p_{d \downarrow}=0$ & - & - & - \\
\hline step 1.1 & $S \in[5,30]$ & northern Sahel & $S C\left(H B R_{a}, E I R_{a}\right)$ & $\Rightarrow S:=10 \mathrm{~mm}$ \\
\hline step 1.2 & $U_{2} \in[500,1000]$ & West Africa & SC(all) & $\Rightarrow U_{2}:=500 \mathrm{~mm}$ \\
\hline step 1.3 & $\# E_{p} \in[50,150]$ & Sahel & $S C\left(H B R_{a}, E I R_{a}\right)$ & $\Rightarrow \# E_{p} \in[75,125]$ \\
\hline step 1.4 & $C A P \in[250,2000]$ & West Africa & SC(all) & $\Rightarrow C A P \in[300,900]$ \\
\hline \multirow[t]{3}{*}{ step 2} & $C A P \in[300,900]$ & West Africa & $S C\left(H B R_{a}, E I R_{a}\right)$ & $\Rightarrow$ CAP: 400 fertile \\
\hline & $\# E_{p} \in[70,130]$ & & & females, $\# E_{p}:=120$ eggs, \\
\hline & $p_{d \downarrow} \in[0,10]$ & & & $p_{d \downarrow}:=-10 \%$ \\
\hline
\end{tabular}

Overview in terms of the evaluation of the performed calibration steps. Columns: step: step number; parameter: particular settings of the model parameter; area: area of interest; SC: applied skill score; result: result of the calibration step (for more details see text). 
of $14^{\circ} 30^{\prime} \mathrm{N}$. This fact impeded a proper determination or a further confinement of $\# E_{p}$. However, the LMM underestimates (overestimates) $E I R_{a}$, when $\# E_{p}$ is set to 50 (150) eggs (see Additional file 3 and step 1.3 in Table 3).

After confirming the parameter range of $\# E_{p}$ (75-125 eggs) also CAP (i.e. the cap on the number of fertile mosquitoes) was more precisely determined (step 1.4 in Table 3). CAP is only of importance for comparatively large annual rainfall amounts. $C A P$ markedly reduces the number of deposited eggs and hence biting rates under wet conditions. In fact, the reduction is markedly pronounced in the Sudanian zone, along the Guinean coast, and in Cameroon for low CAP values $(\leq 750$ fertile females). In contrast, large values of CAP ( $\geq 1000)$ cause fairly high numbers of non-infectious and infectious mosquito bites [11]. The ranking relative to $S C$ (all) shows that LMM versions reveal a small skill when $C A P$ is set to 250 fertile females [11].

\section{Second step}

The basis of the second iteration of the LMM calibration are the conclusions taken from the first step. For this reason, only the setting of $C A P, \# E_{p}$, and $p_{d \downarrow}$ needed to be varied. For the second set of runs, the $\# E_{p}$ values were varied between 70 and 130 eggs (7 steps at increments of 10 eggs) and CAP (i.e. the cap on the number of fertile females) was set between 300 and 900 fertile females (13 steps at 50 fertile females). Five different values for $p_{d \downarrow}$ (i.e. the reduction of dry season mosquito survival) were in addition utilised $(0.0,-2.5,-5.0,-7.5$, and $-10.0 \%)$. The second set of model runs included altogether 455 (originating from the combination of $7 \cdot 13.5$ settings) different model sets of parameter settings.

The second set of runs shows that low (high) $\# E_{p}$ and high (low) $C A P$ values produce the highest skill scores in terms of the eleven variables $(S C(a l l))$. As stated above this is because \#E $E_{p}$ and $C A P$ tend to compensate each other at the more humid locations (see Additional file 3). Particularly notable is the fact that various model versions exhibited comparatively high skill scores. This fact makes a final objective setting of the remaining parameters difficult. For simplicity, the model version with the highest skill score in terms of $H B R_{a}$ and $E I R_{a}$ $\left(S C\left(H B R_{a}, E I R_{a}\right)\right)$ was chosen.

The set of parameter settings with $\# E_{p}=120$ eggs, $C A P=400$ fertile females, and $p_{d \downarrow}=-10 \%$ produced the highest skill score in terms of $H B R_{a}$ and $E I R_{a}$. A total of 78 from 106 possible points (73.6\%) was reached by this model version. With regard to $S C($ all $)$ altogether 279 from the 440 possible points were gained (Additional file 3).

The $\# E_{p}$ value of 120 eggs is in the middle of observations (see Additional file One of Ermert et al. [10]). The $p_{d \downarrow}$ value of $-10 \%$ enables the simulation of a realistic end of the malaria season. Malaria transmission stops one to two months earlier in the model when this $p_{d \downarrow}$ value is applied (cf. Figure 4). The correspondence with MARA (mapping Malaria Risk in Afrika project) maps [43] is improved by the earlier break of malaria transmission.

\section{Validation of the $\mathrm{LMM}_{2010}$}

Based on weather conditions observed at meteorological stations, results of the LMM simulations using the final set of parameter settings (see Table 1) compare well to observed values of eight entomological variables. The new model version leads to the simulation of about the same $E I R_{a}$ values as observed (Figure $3 ; S C\left(E I R_{a}\right)=41$ (54)) in both epidemic and endemic malaria areas of West Africa. For example, the LMM achieves 15 from 17 achieveable points with regard to criterion 1 (enclosure), all points in terms of criterion 3 (median enclosure), and 6 out of 8 points regarding criterion 4 (median quartile enclosure). The performance of the model is lower in terms of criterion 2 (enclosure), which appears with criterion 4 (median quartile enclosure) to be the most rigorous criterion.

Low $E I R_{a}$ values are modelled under dry conditions in the Sahel under epidemic malaria conditions. The $E I R_{a}$ values are much higher for annual rainfall of about $1000 \mathrm{~mm}$ and again decrease as observed in the field data when the model is subject to higher annual rainfall (Figure 3). Within endemic malaria areas the $\mathrm{LMM}_{2010}$ simulation encompasses in most cases the observed values of $E I R_{a}$. For some stations with numerous observations even the median values of $E I R_{a}$ are comparable, for example, in vicinity of Bobo-Dioulasso in Burkina Faso (13 observations) or in the area of Kaolack in Senegal (six observations). However, there are also some exceptions, for example, in vicinity of Barkedji in Senegal the simulated $E I R_{a}$ is much lower than two observations [44]. The high biting rates in this area are probably a result of special local environmental conditions. LeMasson et al. [44] and Molez et al. [45] conjectured that the presence of clay hollows, which collect water as soon as the rains start, caused a long persistence of temporary ponds and hence of malaria transmission. With regard to the annual human biting rate $\left(H B R_{a}\right)$ and consequently also for the annual mean of the circumsporozoite protein rate $\left(C S P R_{a}\right)$, similar statements are valid. There is a correspondence between the $\mathrm{LMM}_{2010}$ simulations of $H B R_{a}$ as well as $C S P R_{a}$ and observations from entomological studies $\left(S C\left(H B R_{a}\right)=37(52) ; S C\left(C S P R_{a}\right)=33\right.$ (55); cf. Additional file 4). At nearly every station at least one observation is enclosed in the simulation (criterion 1; see Table 4). In only two cases the observed 
Table $4 \mathrm{LMM}_{2004}$ and $\mathrm{LMM}_{2010}$ performance

\begin{tabular}{|c|c|c|c|c|c|c|c|c|c|c|c|c|c|c|}
\hline \multirow[b]{2}{*}{$S C(x)$} & \multicolumn{10}{|c|}{ criteria number (see Table 2) } & \multicolumn{4}{|c|}{ criteria number (see Table 2) } \\
\hline & 1 & 2 & 3 & 4 & 5 & 6 & $\mathrm{LMM}_{2004}$ & 1 & 2 & 3 & 4 & 5 & 6 & $\mathrm{LMM}_{2010}$ \\
\hline$S C\left(H B R_{a}\right)$ & $7(16)$ & $5(16)$ & $5(13)$ & $0(7)$ & -60 & - & $-43(52)$ & $14(16)$ & $8(16)$ & $11(13)$ & $4(7)$ & 0 & - & $\mathbf{3 7}(52)$ \\
\hline$S C\left(C S P R_{a}\right)$ & $6(18)$ & $0(16)$ & $2(14)$ & $1(7)$ & - & - & $9(55)$ & $14(18)$ & $4(16)$ & $12(14)$ & $3(7)$ & - & - & $33(55)$ \\
\hline$S C\left(E I R_{a}\right)$ & $12(17)$ & $7(16)$ & 10(13) & $1(8)$ & -50 & - & $-20(54)$ & $15(17)$ & $7(16)$ & $13(13)$ & $6(8)$ & 0 & - & $41(54)$ \\
\hline$\Sigma$ & $25(51)$ & $12(48)$ & $17(40)$ & $2(22)$ & -110 & - & $-54(161)$ & $43(51)$ & $19(48)$ & $36(40)$ & $13(22)$ & 0 & - & 111(161) \\
\hline SC(Seas) & $11(16)$ & $5(12)$ & $7(9)$ & $4(5)$ & - & - & $27(42)$ & $15(16)$ & $4(12)$ & $8(9)$ & $4(5)$ & - & - & $31(42)$ \\
\hline SC(MSeas) & $13(14)$ & $5(12)$ & $4(7)$ & $0(4)$ & - & - & $22(37)$ & $12(14)$ & $9(12)$ & $7(7)$ & $O(4)$ & - & - & 28(37) \\
\hline SC(XSeas) & $12(17)$ & $4(14)$ & - & - & - & $2(10)$ & $18(41)$ & $15(17)$ & $6(14)$ & - & - & - & $2(10)$ & $23(41)$ \\
\hline SC(SSeas) & $11(17)$ & $2(14)$ & - & - & - & $3(10)$ & $16(41)$ & $16(17)$ & $7(14)$ & - & - & - & $5(10)$ & $\mathbf{2 8}(41)$ \\
\hline SC(ESeas) & $15(16)$ & $3(12)$ & - & - & - & $4(9)$ & $22(37)$ & 13(16) & $3(12)$ & - & - & - & 2(9) & 18(37) \\
\hline$\Sigma$ & $62(80)$ & 19(64) & $11(16)$ & 4(9) & - & $9(29)$ & 105(198) & $71(80)$ & $29(64)$ & $15(16)$ & $4(9)$ & - & $9(29)$ & $128(198)$ \\
\hline$S C\left(P R_{a}\right)$ & $4(13)$ & 1(9) & $2(5)$ & $1(2)$ & - & - & $8(29)$ & $9(13)$ & $3(9)$ & $3(5)$ & 1(2) & - & - & 16(29) \\
\hline$S C\left(P R_{\max }, a\right)$ & $7(11)$ & $2(7)$ & $3(5)$ & $O(2)$ & - & - & $12(25)$ & $8(11)$ & $4(7)$ & $4(5)$ & $O(2)$ & - & - & $16(25)$ \\
\hline$S C\left(P R_{\min , a}\right)$ & $3(12)$ & $1(8)$ & $0(5)$ & $O(2)$ & - & - & 4(27) & $5(12)$ & $1(8)$ & $2(5)$ & $O(2)$ & - & - & $8(27)$ \\
\hline$\Sigma$ & 14(36) & $4(24)$ & $5(15)$ & 1(6) & - & - & $24(81)$ & $22(36)$ & $8(24)$ & $9(15)$ & $1(6)$ & - & - & $40(81)$ \\
\hline SC(all) & $101(167)$ & $35(136)$ & $33(71)$ & $7(37)$ & -110 & $9(29)$ & $75(440)$ & $136(167)$ & $56(136)$ & $60(71)$ & $18(37)$ & 0 & $9(29)$ & $279(440)$ \\
\hline
\end{tabular}

Performance of $\mathrm{LMM}_{2004}$ and $\mathrm{LMM}_{2010}$ relative to entomological and parasitological field studies in West Africa. Digits are taken in bold, when either the LMM 2004 or the LMM $\mathrm{LM}_{2010}$ performs better. Values in italics indicate no difference between the two model versions. The numbers in brackets refer to points that could be theoretically achieved (see text). Inserted are the scores of the $\mathrm{LMM}_{2004}$ and $\mathrm{LMM}_{2010}$ in terms of single criteria (see Table 2) as well as to their sum (columns: $\mathrm{LMM}_{2004}$ and $\mathrm{LMM}_{2010}$ ). Columns: $S C(x)$ : skill score with regard to variable $x ; 1$ : score regarding the first criterion (overlap); 2 : enclosure; 3 : median enclosure; 4: median quartile enclosure; 5: penalty; 6: frequency. Subtotals $(\Sigma)$ are furthermore inserted in terms of the skill scores of entomological $\left(H B R_{a}\right.$, CSPR $R_{a}$ and $\left.E I R_{a}\right)$, malaria seasonality (Seas, MSeas, XSeas, SSeas, and ESeas), as well as parasitological variables $\left(P R_{a}, P R_{\max }, a\right.$, and $P R_{\min }$ a). The comparison of the skill scores of the eleven malaria variables with regard to the performance of the $\mathrm{LMM}_{2004}$ and $\mathrm{LMM}_{2010}$ (including only values from columns: $L M M M_{2004}$ and $L M M_{2010}$ ) reveals a $P$ value of 0.0064 in terms of the Wilcoxon signed rank test.

median is not enclosed in the LMM simulations (criterion 3; Table 4).

Another result of the calibration is that the simulations capture the variability of malaria transmission. The interannual variability of $E I R_{a}$, for example, is fairly large. For most stations the number of infectious mosquito bites fluctuates between values of less than 100 and several hundred. Unfortunately, long-term studies are rare and continuous observations from rural sites are only available from Ndiop for four years (Senegal; $\left.13^{\circ} 41^{\prime} \mathrm{N}, 16^{\circ} 23^{\prime} \mathrm{W}\right)$. In this Sahelian village, malaria transmission varied in the mid of the $1990 \mathrm{~s}$ between seven and 63 infective bites per human per year [34]. For this area, simulated $E_{1} R_{a}$ values range from almost zero to about 158 infectious bites per human per year. Note that the LMM simulation refers to the meteorological data from Kaolack, which is located about $55 \mathrm{~km}$ to the northeast of Ndiop.

The simulation of the malaria seasonality by the $\mathrm{LMM}_{2010}$ is consistent with epidemic and endemic field observations, despite the fact that a part of the field observations reveals considerably heterogeneous values. The simulated length of the season agrees well with the observations $(S C($ Seas $)=31(42))$. Only in one out of 16 cases is there no overlap (criterion 1) with observations. In three out of the five cases with at least five field observations, the optimum scoring of four points is achieved (see Additional file 4). In general, the season length shortens with decreasing annual rainfall. Short malaria seasons and no malaria transmission are simulated for the Sahel and year-round transmission is found, for instance, in Cameroon. Also the length of the main transmission season corresponds roughly to the observations $(S C($ MSeas $)=28(37))$. At only two of the 14 weather stations no overlappings (criterion 1) are found. Criterion 3 (median enclosure) is fulfilled for each of the seven cases (Table 4).

The skill score in terms of the maximum transmission month (XSeaS) gains 23 from the 41 achieveable points $(S C($ XSeas $)=23(41)$; cf. Additional file 4$)$. The month of maximum transmission overlaps (criterion 1) between simulations and observations in 15 out of 17 cases and all observations are enclosed in the simulation range (criterion 2) in six out of 16 cases. The simulated month with the maximum transmission therefore corresponds frequently with that of the observations, especially in the Sahel. However, in terms of XSeas, the model simulations disagree in Cameroon, at the weather stations of Yaoundé and Koundja for areas with yearround endemic malaria transmission.

Both the start (SSeas) and end (ESeas) of the malaria season are realistically reproduced by the $\mathrm{LMM}_{2010}$ version $($ Figure 4; SC(SSeas $)=28(41) ; S C($ ESeas $)=18(37))$. With regard to SSeas the observations and simulations 
do not overlap only in one case (criterion 1; Table 4). In terms of ESeas, in 5 out of 10 cases the maximum number of observations and simulations are found in the same month (criterion 6; Table 4). For weather stations that observed no malaria transmission in most years, the model also simulates most frequently no transmission. The same is true for Yaoundé; in terms of year-round transmission (see Additional file 4). Observations that fall outside of simulations hardly differ more than one month.

The performance of the $\mathrm{LMM}_{2010}$ with regard to the annual mean $\left(P R_{a}\right)$, annual minimum $\left(P R_{\text {min, } a}\right)$ and annual maximum of the asexual parasite ratio $\left(P R_{\max , a}\right)$ is somewhat mixed. The main reason for the relatively low skill scores is the heterogeneous parasitological observations that cannot be reproduced by the LMM (Figure 5 and Additional file 4). Measurements of the $P R_{\text {min }}$, for example, exhibit a remarkable spread. Nine observations of $P R_{\text {min }}$ a reveal higher values than $50 \%$ whereas 23 values are lower than this threshold. These differences suggest that some special factors are strongly affecting $P R$ values. As a consequence, the $\mathrm{LMM}_{2010}$ reaches only eight from 27 possible points (Figure 5; Table 4). Due to the fact that there is a stronger yearto-year variability in the $\mathrm{LMM}_{2010}$ in terms of the simulation of $P R_{a}$ and $P R_{\max , a}$ the model performs better with regard to these two parasitological variables (SC $\left(P R_{a}\right)=16$ (29); $S C(P R \max ; a)=16$ (25); Table 4; Additional file 4$)$.

\section{$\mathrm{LMM}_{2010}$ versus $\mathrm{LMM}_{2004}$}

In this section, the performance of the LMM version of 2004 (henceforth called $\mathrm{LMM}_{2004}$ ) is compared to that of the $\mathrm{LMM}_{2010}$. The LMM is validated using simulations that are based on time series driven by weather station observations (see Table 4). In addition, gridded $\mathrm{LMM}_{2004}$ and $\mathrm{LMM}_{2010}$ runs driven by the biascorrected REMO data (see data sources) are compared (Figure 6).

The validation of the $\mathrm{LMM}_{2004}$ and $\mathrm{LMM}_{2010}$ runs based on weather station data from West Africa clearly demonstrates a significant improvement of the original model version (the $P$ value is 0.0064 ; see Table 4 ). Except for ESeas, all skill scores are higher for the $\mathrm{LMM}_{2010}$ than for the $\mathrm{LMM}_{2004}$. Comparison of the $\mathrm{LMM}_{2004}$ simulations with field observations reveal two general features: (i) The $\mathrm{LMM}_{2004}$ fails to simulate malaria transmission in various malarious semi-arid regions and significantly overestimates the transmission in humid areas especially regions with endemic malaria territories. Note here that the $\mathrm{LMM}_{2004}$ was not designed to be used in endemic areas. (ii) Values of the parasite ratio, expressed in the three parasitological variables, are not optimal in the original model version. In terms of ESeas, the improvement of the model, especially in the Sahel, seems not to show up due to the lack of numerous observations of ESeas in this area. Nevertheless, the $\mathrm{LMM}_{2010}$ represents a significant step forward in the modelling of a weather-driven malaria transmission cycle.

The first feature (i) is a result of the simplified linear relationship between rainfall and the deposition of eggs by female mosquitoes in the $\mathrm{LMM}_{2004}$. In predominantly dry areas the model is not able to produce a reasonable size of the mosquito population. The $\mathrm{LMM}_{2004}$ hence fails to simulate malaria transmission in the northern part of the Sahel (Figure 6a \&6c). By contrast, in the $\mathrm{LMM}_{2010}$ runs, malaria transmission is found as far north as about $18^{\circ} \mathrm{N}$ (Figure $6 \mathrm{~b} \& 6 \mathrm{~d}$ ). This seems to be realistic since, for example, a definite malaria season was observed south of Agadez [46], which is located at about $17^{\circ} \mathrm{N}$. Further the MARA project detected epidemic-prone areas up to $20^{\circ} \mathrm{N}$ [47].

In the second step, LMM runs were driven by gridded and bias-corrected REMO data for 1960-2000. Based on this input data the $\mathrm{LMM}_{2010}$ seems to reproduce a realistic picture with regard to transmission rates and the malaria seasonality for the whole of Africa including epidemic and endemic malaria areas (cf. Figure 6b \&6d). This is in contrast to the $\mathrm{LMM}_{2004}$ which was originally only constructed for epidemic malaria territories [9]. This former model version simulates a tremendous number of mosquitoes and generates significantly too high $E I R_{a}$ values for humid areas such as the equatorial tropics (Figure 6a). $H B R_{a}$ partly exceeds 1,000,000 bites per annum in these endemic malaria areas [11]. Note that $H B R_{a}$ field values are usually much lower than 50,000 bites (see Additional file 2). However, the original model version also reveals deficiencies in terms of the spread of malaria within epidemic regions. The $\mathrm{LMM}_{2004}$ fails to simulate malarious areas north of about $16^{\circ} \mathrm{N}$.

The infectiousness of mosquitoes is underestimated by the $\mathrm{LMM}_{2004}$ since values of the circumsporozoite protein rate (here $C S P R_{a}$ ) are in general lower than 1\% [11] resulting from the comparatively low mosquito survival of the Martens I scheme (see Figure 3 in [10]). In addition, the start of the malaria season is notably delayed in the $\mathrm{LMM}_{2004}$ simulations. The start of the season occurs about one to two months later under the original than under the new LMM version [11]. The strong growth of the mosquito population causes shorter main transmission seasons (MSeas values) in the $\mathrm{LMM}_{2004}$ runs than in that of the $\mathrm{LMM}_{2010}$ [11]. Moreover, a later occurrence of XSeas is found for the $\mathrm{LMM}_{2004}$ simulations.

Deficiencies of the $\mathrm{LMM}_{2004}$ are also found for the three parasitological variables. Almost the whole 


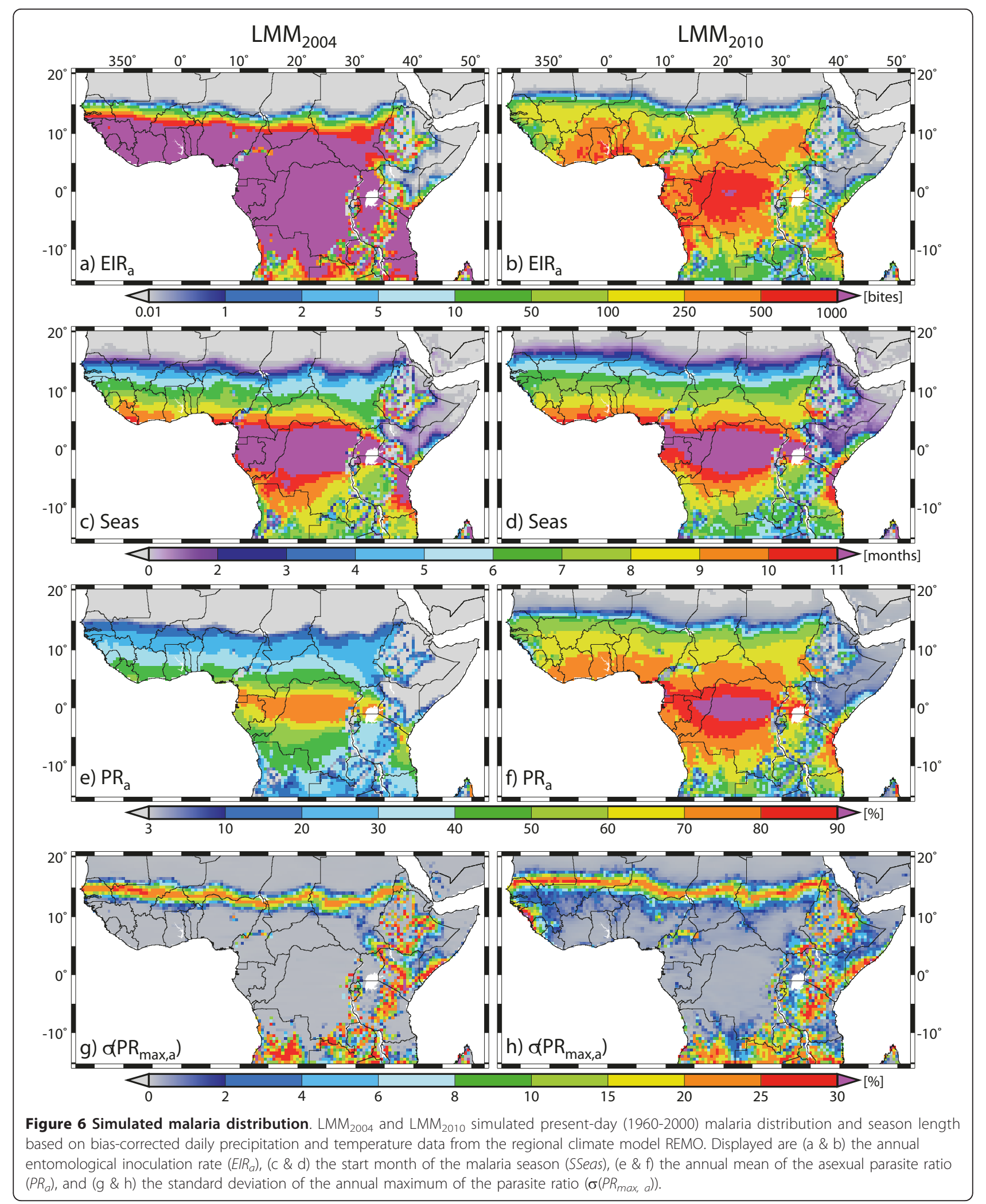


population clears the malaria parasite during the dry season due to the high recovery rate $(r)$ of the $\mathrm{LMM}_{2004}$ $\left(\mathrm{LMM}_{2004}\left(\mathrm{LMM}_{2010}\right): r=0.0284(0.005) \mathrm{day}^{-1}\right.$; see [10]). Such a characteristic is, however, not found in parasitological surveys (see Figure 5) and results in a very low skill in terms of $P R_{\text {min }}$ a (see Table 4). The strong recovery from infection, moreover, underestimates the values of $P R_{a}$ and $P R_{\max }, a$ [11]. In comparison to $\mathrm{LMM}_{2010}$ runs, parasite ratios are hence much lower in the $\mathrm{LMM}_{2004}$ simulations (see Figure 6e \&6f).

One characteristic of malaria epidemic areas is the sudden and unexpected increase of the parasite ratio in certain years. Epidemic regions therefore should reveal a strong interannual variability of the annual maximum of the asexual parasite ratio $\left(P R_{\max }\right.$, a Figure $6 \mathrm{~g} \& 6 \mathrm{~h})$ and in general low annual mean asexual parasite ratios $\left(P R_{a}\right.$; Figure $\left.6 \mathrm{e} \& 6 \mathrm{f}\right)$. According to LMM simulations forced with gridded REMO data, epidemic areas are found along a strip within the Sahelian zone as well as for highland territories and fairly dry areas in East Africa, Zambia, and Angola (Figure 6g \&6h). Most notable is the fact that in the $\mathrm{LMM}_{2004}$ runs the strip of high epidemic risk is detected about $2-4^{\circ}$ to the south in comparison to that of the $\mathrm{LMM}_{2010}$. Note that the $\mathrm{LMM}_{2010}$ simulations seem to be more realistic in comparison to previous assessments of epidemic territories in the Sahel [47]. Differences between $\mathrm{LMM}_{2004}$ and $\mathrm{LMM}_{2010}$ simulations are also found outside of the Sahelian zone. Overall the $\mathrm{LMM}_{2010}$ seems to result in a stronger interannual variability of $P R_{\max }, a$.

\section{Discussion}

The aim of the present study was the development of an improved weather-driven malaria model, which is able to simulate malaria transmission in both epidemic as well as endemic malaria areas. This section provides a detailed discussion with regard to various aspects of the present study. The present-day climate performance of the new model version are discussed relative to results of former studies and the model calibration is evaluated.

Comparison of $\mathrm{LMM}_{2010}$ runs with those performed by the original formulation of the model reveals a significant improvement (see Table 4) of the model performance both for epidemic as well as endemic malaria areas. In contrast to the $\mathrm{LMM}_{2004}$, the $\mathrm{LMM}_{2010}$ is able to reproduce the low transmission rates in the northern part of the Sahel. This enables, therefore, an improved detection of epidemic areas, in particular, in the Sahel. The $\mathrm{LMM}_{2010}$ validation also shows that the model can now also be applied for endemic malaria areas. The usage of the fuzzy distribution model enables the simulation of realistic sizes of the mosquito population under humid rainfall conditions resulting in reasonable transmission rates. Moreover, the lag in the malaria seasonality has disappeared in the new version of the LMM.

Various published malaria distribution maps [43,48] correspond well with the simulated spread of malaria by the $\mathrm{LMM}_{2010}$. However, in certain parts the simulated intensity of malaria transmission differs considerably from published malaria maps. The $\mathrm{LMM}_{2010}$ in general seems to predict higher transmission rates than satellitederived predictions of $E I R_{a}$ from Rogers et al. [48]. The maps of transmission intensity provided by Gemperli et al. [49] are fairly patchy. In fact, the prediction from Gemperli et al. significantly suffers from the neglected interannual variability of malaria. Based on the few available $E I R_{a}$ observations it is difficult to judge which estimates are closer to reality. However, the validation of the $\mathrm{LMM}_{2010}$ under different climatic conditions provides evidence that the present study generated realistic biting rates and a reasonable interannual variability.

The calibration of the LMM was performed in West Africa for different atmospheric conditions of epidemic and endemic malaria regions. Realistic temperature and precipitation time series were reconstructed from various synoptic weather stations. The comparison with observations from eleven entomological and parasitological variables finally enabled the setting of undetermined model parameters.

The databases (including meteorological, entomological, and parasitological observations) for the LMM calibration are not optimal. There is a mismatch between the scales at which a disease vector responds to hydrologic variability and the scales at which hydrologic variability is actually observed. Systems should be developed that monitor hydrologic variability at scales corresponding to disease system ecologies [50]. In this study, the generation of realistic meteorological station time series enabled the comparison with atmospheric conditions from malaria field studies, which were not conducted directly at the weather stations. These sites therefore in any case exhibit a different temporal variability of rainfall and temperatures. This might be one reason, amongst other factors such as environmental conditions, why year-to-year comparisons between observation and simulation were weakly correlated at single locations. In order to circumvent this problem, the present study refrained from looking at paired annual correlations at single stations but applied a problem-adapted scoring system.

The required historical entomological and parasitological data are rarely available with sufficient coverage. Most locations show only one, two, or even no field measurements. It is therefore likely that a larger set of observations would have an impact on the result of the model calibration. Ideally, model simulations and 
malaria observations should be compared from year-toyear. However, this would require the simultaneous monitoring of long-term malaria data and meteorological measurements. Such long time series are available for the area of Ndiop/Senegal (S. Louvet, personal communication, 2007), but these data sets are not publicly available.

The close ranking of diverse model runs as well as the lack of sufficient validation data further restricted an objective formal fitting of the model. In fact, various steps of the calibration procedure were subjective. Due to high computational costs it was furthermore not possible to fit all remaining model parameters simultaneously. However, because various settings compensate each other it is likely that the final model formulation conforms as much as possible to reality.

The calibration and validation of the model should also be ideally not only restricted to West Africa. However, such an extension to, for example, East Africa was beyond the scope of this study. The Malaria Atlas Project intends to provide access to various malaria studies [51]. This might provide an efficient access to malaria data beyond that of West Africa. Such an extension would ideally include East African highlands and an estimation of the sporogonic temperature threshold.

This study was naturally not able to account for all processes involved in the spread of malaria. Some factors might be included in a future extension of the LMM. The simulation of the parasitological malaria variables by the $\mathrm{LMM}_{2010}$ is a simplification of real processes. The validation of the $\mathrm{LMM}_{2010}$ by means of parasitological measurements in West Africa revealed shortcomings of the new model version. Lower skill scores were achieved by the three parasitological variables when compared to the results from the eight entomological variables.

In addition to the lack of immunity, the $\mathrm{LMM}_{2010}$ does not account for other malaria factors such as chemoprophylaxis and human activities. However, this could be implemented by means of a variable parameter setting. Observations suggest a greater variability of the parasite ratio. At Bobo-Dioulasso, for example, the ten observed annual mean asexual parasite ratios $\left(P R_{a}\right)$ range between 29.1 and $77.5 \%$. In contrast, the 34 annual values of the $\mathrm{LMM}_{2010}$ only span values between 50 and $70 \%$ [11].

Due to the lack of long-term observations, Kleinschmidt et al. [52] and Gemperli et al. [49] were forced to neglect the interannual variability of $P R$. This fact might again partly be responsible for their projected irregular $P R$ pattern in West Africa. Their maps also show a sharp decrease of $P R_{a}$ north of about $15^{\circ} \mathrm{N}$. In contrast to the $\mathrm{LMM}_{2010}$ runs and the Garki model simulations from Gemperli et al. [49], $P R_{a}$ is frequently lower than
$50 \%$ south of $15^{\circ} \mathrm{N}$. Only few regions exhibit higher $P R_{a}$ values than $70 \%$, which are simulated by the $\mathrm{LMM}_{2010}$.

It should be pointed out here that climate is rarely the only important driver of malaria. Numerous other studies showed $[21,22,53,54]$ that in particular human activities are crucial for the transmission and prevention of malaria across Africa. For example, the modification of the landscape by irrigation [55], forest clearing [56], or urbanization [57] can significantly alter malaria transmission. The present study assessed only the malaria risk for rural areas without the influence of permanent breeding places. The applicability of this analysis is therefore limited when permanent water bodies or urban centres are present. In principle, the calibration of the $\mathrm{LMM}_{2010}$ could also be performed for urban areas. However, such an undertaking seems to be hampered by the lower number of available observations (see Additional file 2).

\section{Conclusions}

One of the most comprehensive studies to date in terms of gathering validation data and information from the literature for the development of a new version of an existing malaria model was conducted for West Africa. The first part of this study [10] provided new parameter settings of the LMM and changed some key processes in the model. The performance of numerous model versions were compared to malaria field observations from rural sites with no malaria measures and irrigation in order to maximise the climate-driven malaria impact. The comparison with observations from eleven entomological and parasitological variables finally enabled the specification of a final set of parameter settings of the model. Validation of the new LMM version in West Africa reveals that the simulations and malaria seasonality compare well with entomological field observations of epidemic and endemic malaria areas. The $\mathrm{LMM}_{2010}$ also demonstrates a fairly realistic simulation of the malaria spread as well as an improved detection of epidemic risk in Africa. Due to model limitations, the performance of the $\mathrm{LMM}_{2010}$ is somewhat weaker with regard to parasitological variables.

It is concluded that the $\mathrm{LMM}_{2010}$ represents a significant step forward in the modelling of a weather-driven malaria transmission cycle. In contrast to the original model, the application of the new model version is not only restricted to epidemic malaria regions but the usability is now extended to endemic malaria areas. Ermert [11] hence used the $\mathrm{LMM}_{2010}$ for the assessment of malaria risk under the influence of observed and projected climate change using regionalized climate projections for Africa. The $\mathrm{LMM}_{2010}$ simulated transmission rates were passed to the Garki model to form a hybrid malaria model, which is able to consider further aspects 
of the malaria disease such as age-dependent parasite ratios as well as the immune status of the population. In the near future, this hybrid model will be applied in a health early warning system, which is implemented by the QWeCI (Quantifiying Weather and Climate Impacts on Health in Developing Countries) project from the European Community's Seventh Framework Research Programme. Further work might enable the inclusion of some other processes involved in the spread of malaria into the LMM.

\section{Additional material}

Additional file 1: Synoptic weather stations. Information relative to synoptic weather stations from West Africa. The country, name, identifier, latitude and longitude positions, as well as the elevation of the meteorological stations are given. The LMM was driven by reconstructed temperature and precipitation time series (1973-2006) from these meteorological stations.

Additional file 2: Entomological and parasitological data. Data with regard to entomological and parasitological observations from malaria field studies.

Additional file 3: Skill scores in terms of the LMM validation. Ranks in terms of skill scores as computed for simulations of different LMM sets of parameter settings: (1) Top 10 and last 5 of 300 malaria runs from the first calibration step according to the skill score of the annual human biting and entomological inoculation rates $\left(S C\left(H B R_{a}, E I R_{a}\right)\right)$. (2) Top 10 of 300 malaria runs from the first calibration step in terms of all eleven entomological and parasitological malaria variables (SC(all)). (3) Top 10 of 455 malaria runs from the second calibration step relative to $S C\left(H B R_{a}\right.$, $\left.E / R_{a}\right)$. (4) Top 10 of 455 malaria runs from the second calibration step regarding $S C($ all $)$

Additional file 4: Observed and simulated entomological and parasitological values of the $\mathrm{LMM}_{2010}$. Validation of $\mathrm{LMM}_{2010}$ simulations in terms of $H B R_{a}, C S P R_{a}$, Seas, MSeas, XSeas, SSeas, PRa, and $P R_{\max , a}$ in the area of the 34 synoptic stations in West Africa as ordered in Figure 2.

\section{List of abbreviations}

DWD: German Weather Service; ECHAM5/MPI-OM: European Centre HAmburg Model, 5th generation/Max-Planck-Institute-Ocean Model; GSOD: Federal climate complex Global Surface Summary of Day version 7; LMM: Liverpool Malaria Model; LMM 2004: Liverpool Malaria Model version of 2004; $\mathrm{LMM}_{2010}$ : Liverpool Malaria Model version of 2010; MARA: Mapping Malaria Risk in Africa;

\section{List of symbols}

$\# E_{p}$ : number of produced eggs per female mosquito; CAP: cap on the number of fertile mosquitoes; CSPR: CircumSporozoite Protein Rate; CSPR: annual mean CircumSporozoite Protein Rate; EIR: Entomological Inoculation Rate; $E I R_{m}$ : monthly Entomological Inoculation Rate; EIRa: annual Entomological Inoculation Rate; ESeas: End month of the malaria Season; HBR: human Biting Rate; $H B R_{a}$ : annual Human Biting Rate; MSeas: length of the Main malaria Season; $p_{d \downarrow}$ : dry season mosquito survival probability shift off; $P R$ : asexual Parasite Ratio; $P R_{a}$ : annual mean asexual Parasite Ratio; $P R_{\text {max }}$ $a$ : annual maximum of the asexual Parasite Ratio; $P R_{\min ,} a$ : annual minimum of the asexual Parasite Ratio; $r$ : recovery rate; $R$ : rainfall; $R_{a}$ : annual rainfall amount; $R_{\Sigma 10 d}$ : 10-day accumulated precipitation; $S$ : most suitable rainfall condition according to the fuzzy distribution model; SC(all): skill score with regard to the used eleven malaria variables; $S C(x)$ : skill score with regard to malaria variable $x$; Seas: length of the malaria Season; SSeas: Start month of the malaria Season; $T$ : temperature; $T_{a}$ : annual mean temperature; $U_{1}$ : lower threshold of unsuitable rainfall conditions with regard to the fuzzy distribution model; $U_{2}$ : upper threshold of unsuitable rainfall conditions regarding the fuzzy distribution model; XSeas: month of maXimum transmission, i.e. the month with the largest EIR value.

\section{Acknowledgements}

We are grateful to numerous people who kindly provided useful data, literature references, and special knowledge for this study. These are: Dipl.Met. C. Baugirdis from DWD/Offenbach (weather station data for West Africa), Dr. S. Louvet from the 'Centre de Recherches de Climatologie (CRC)'/ Dijon (malaria literature), as well as S. Stephens and N. Lott from US National Climatic Data Center (interpretation of GSOD precipitation).

This study was part of the IMPETUS West Africa project and was supported by the Federal German Ministry of Education and Research (BMBF) under grant No. 01 LW06001A in the GLOWA programme and by the Ministry of Innovation, Science, Research, and Technology (MIWFT) of the federal state of North Rhine-Westphalia under grant No. 313-21200200. The support by the EU project AMMA (funded by the European Commission's Sixth Framework Research Programme through project No. 004089) and the Natural Environment Research Council (NERC) AMMA is gratefully acknowledged by AEJ and APM. Based on a French initiative, AMMA was built by an international scientific group and was funded by a large number of agencies, especially from France, United Kingdom, United States, and Africa. Detailed information on scientific coordination and funding is available on the AMMA international website http://www.ammainternational.org. Finally, we wish to thank one anonymous reviewer whose comments helped to greatly improve the manuscript.

\section{Author details}

${ }^{1}$ Institute of Geophysics and Meteorology, University of Cologne, Cologne, Germany. ${ }^{2}$ School of Environmental Sciences, University of Liverpool, Liverpool, UK.

\section{Authors' contributions}

VE designed the study, undertook the literature review, proposed changes in the mathematical formulation of the LMM, ran the model, analysed the data from the model runs, and wrote the manuscript. AHF and APM contributed to the concept of the study. AHF supervised the PhD study of VE whose results formed the basis of the manuscript. APM was furthermore originally involved in the formulation of the LMM. AEJ contributed to the new design of LMM and provided the new model code. All authors read, suggested changes, and approved the final manuscript.

\section{Competing interests}

The authors declare that they have no competing interests.

Received: 17 September 2010 Accepted: 16 March 2011 Published: 16 March 2011

\section{References}

1. WHO: World malaria situation in 1994. Wkly Epidemiol Rec 1997, 72:269-276.

2. Greenwood B, Mutabingwa T: Malaria in 2002. Nature 2002, 415:670-672.

3. Rogers DJ, Randolph SE: The global spread of malaria in a future, warmer world. Science 2000, 289:1763-1766, (Corrected in print, 29 Sept. 2000, p. 2283).

4. Confalonieri U, Menne B, Akhtar R, Ebi KL, Hauengue M, Kovats RS, Revich B, Woodward A: Human health. In Climate change 2007: impacts, adaptation and vulnerability. Edited by: Parry ML, Canziani OF, Palutikof JP, van der Linden PJ, Hanson CE. Cambridge, United Kingdom: Cambridge University Press; 2007:391-431, [Contribution of working group II to the fourth assessment report of the Intergovernmental Panel on Climate Change].

5. Jones AE, Morse AP: Application and validation of a seasonal ensemble prediction system using a dynamic malaria model. J Clim 2010, 23:4202-4215.

6. Patz JA, Campbell-Lendrum D, Holloway T, Foley JA: Impact of regional climate change on human health. Nature 2005, 438:310-317.

7. Tompkins AM, Feudale L: Seasonal ensemble predictions ofWest African monsoon precipitation in the ECMWF system 3 with a focus on the AMMA special observing period in 2006. Weather and Forecasting 2010, 25:768-788. 
8. Solomon S, Qin D, Manning M, Chen Z, Marquis M, Averyt KB, Tignor M, Miller HL: Climate change 2007: the physical science basis Cambridge, United Kingdom and New York, USA: Cambridge University Press; 2007 [Contribution of working group I to the fourth assessment report of the Intergovernmental Panel on Climate Change].

9. Hoshen $M B$, Morse AP: A weather-driven model of malaria transmission. Malar J 2004, 3:32

10. Ermert $V$, Fink $A H$, Jones $A E$, Morse AP: A new version of the Liverpool Malaria Model. I. Review of the parameter setting and model structures. Malar J. 2011, 10(1):35.

11. Ermert $\mathrm{V}$ : Risk assessment with regard to the occurrence of malaria in Africa under the influence of observed and projected climate change. PhD thesis University of Cologne, Cologne, Germany; 2010 [http://kups.ub. uni-koeln.de/volltexte/2010/3109/].

12. Faye O, Fontenille D, Herve JP, Diack PA, Mouchet SDJ: Le paludisme en zone sahelienne du Sénégal. 1. Données entomologiques sur la transmission. Ann Soc Belg Med Trop 1993, 73:21-30.

13. Quakyi IA, Leke RGF, Befidi-Mengue R, Tsafack M, Bomba-Nkolo D, Manga L, Tchinda V, Njeungue E, Kouontchou S, Fogako J, Nyonglema P, Harun LT, Djokam R, Sama G, Eno A, Megnekou R, Metenou S, Ndountse L, SameEkobo A, Alake G, Meli J, Ngu J, Tietche F, Lohoue J, Mvondo JL, Wansi E, Leke R, Folefack A, Bigoga J, Bomba-Nkolo C, Titanji V, Walker-Abbey A, Hickey MA, Johnson AH, Taylor DW: The epidemiology of Plasmodium falciparum malaria in two Cameroonian villages: Simbok and Etoa. Am J Trop Med Hyg 2000, 63:222-230.

14. Detinova TS: Age-grouping methods in Diptera of medical importance with special reference to some vectors of malaria. No. 47 in Monograph Series, WHO 1962.

15. Paeth $H$, Born $K$, Girmes $R$, Podzun $R$, Jacob D: Regional climate change in Tropical and Northern Africa due to greenhouse forcing and land use changes. J Clim 2009, 22:114-132.

16. Jacob D, van den Hurk BJJM, Andrae U, Elgered G, Fortelius C, Graham LP, Jackson SD, Karstens U, Koepken C, Lindau R, Podzun R, Rockel B, Rubel F, Sass BH, Smith R, Yang $X$ : A comprehensive model intercomparison study investigating the water budget during the PIDCAP period. Meteorology and Atmospheric Physics 2001, 77:19-44.

17. Roeckner E: IPCC-AR4 MPI-ECHAM5 T63L31 MPI-OM GR1.5L40 20C3 M run no.2: atmosphere 6 HOUR values MPImet/MaD Germany. 2004 [http://cera-www.dkrz.de/WDCC/ui/Compact.jsp?acronym=EH5-T63L31_OMGR1.5L40_20C_2_6H], World data center for climate. CERA-DB "EH5-T63L31 OM-GR1.5L40 20C 2 6H".

18. Roeckner E, Lautenschlager M, Schneider H: IPCC-AR4 MPI-ECHAM5 T63L31 MPI-OM GR1.5L40 20C3 M run no.1: atmosphere 6 HOUR values MPImet/MaD Germany. 2006, World data center for climate.

19. Roeckner E, Lautenschlager M, Schneider H: IPCC-AR4 MPI-ECHAM5 T63L31 MPI-OM GR1.5L40 20C3 M run no.3: atmosphere 6 HOUR values MPImet/MaD Germany. 2006, World data center for climate.

20. Speth $P$, Christoph M, Diekkrüger B, Bollig M, Fink AH, Goldbach H, Heckelei T, Menz G, Reichert B, Rössler M: Impacts of global change on the hydrological cycle in West and Northwest Africa Heidelberg, Germany: Springer; 2010 [http://www.springer.com/978-3-642-12956-8].

21. Hay SI, Guerra CA, Tatem AJ, Atkinson PM, Snow RW: Urbanization, malaria transmission and disease burden in Africa. Nat Rev Microbiol 2005, 3:81-90.

22. Kelly-Hope LA, McKenzie FE: The multiplicity of malaria transmission: a review of entomological inoculation rate measurements and methods across sub-Saharan Africa. Malar J 2009, 8:19.

23. Carnevale P, Guillet P, Robert V, Fontenille D, Doannio J, Coosemans M, Mouchet J: Diversity of malaria in rice growing areas of the Afrotropical region. Parassitologia 1999, 41:273-276.

24. Keiser J, Castro MCD, Maltese MF, Bos R, Tanner M, Singer BH, Utzinger J: Effect of irrigation and large dams on the burden of malaria on a global and regional scale. Am J Trop Med Hyg 2005, 74:392-406.

25. Konaté L, Diop A, Sy N, Faye MN, Deng Y, Izri A, Faye O, Mouchet J: Comeback of Anopheles funestus in Sahelian Senegal. Lancet 2001, 358:336.

26. Ijumba JN, Shenton FC, Clarke SE, Mosha FW, Lindsay SW: Irrigated crop production is associated with less malaria than traditional agricultural practices in Tanzania. Trans R Soc Trop Med Hyg 2002, 96:476-480.

27. Lindsay SW, Alonso PL, Schellenberg JRMA, Hemingway J, Thomas PJ, Shenton FC, Greenwood BM: A malaria control trial using insecticide- treated bed nets and targeted chemoprophylaxis in a rural area of The Gambia, West Africa: 3. entomological characteristics of the study area. Trans R Soc Trop Med Hyg 1993, 87(Suppl. 2):19-23.

28. Lindsay SW, Alonso PL, Schellenberg JRMA, Hemingway J, Adiamah JH, Shenton FC, Jawara M, Greenwood BM: A malaria control trial using insecticide-treated bed nets and targeted chemoprophylaxis in a rural area of The Gambia, West Africa: 7. impact of permethrin-impregnated bed nets on malaria vectors. Trans R Soc Trop Med Hyg 1993, 87(Suppl. 2):45-51.

29. Akogbéto $M$, Nahum $A$ : Impact des moustiquaires imprégnées de deltaméthrine sur la transmission du paludisme dans un milieu côtier lagunaire, Bènin. Bull Soc Pathol Exot 1996, 89:291-298.

30. Cuzin-Ouattara N, den Broek AHV, Habluetzel A, Diabate A, SanogoIlboudo E, Diallo DA, Cousens SN, Esposito F: Wide-scale installation of insecticide-treated curtains confers high levels of protection against malaria transmission in a hyperendemic area of Burkina Faso. Trans $R$ Soc Trop Med Hyg 1999, 93:473-479.

31. Le Goff G, Robert V, Fondjo E, Carnevale P: Efficacy of insecticide impregnated bed-nets to control malaria in a rural forested area in southern Cameroon. Mem Inst Oswaldo Cruz 1992, 87(Suppl. 3):355-359.

32. Le Goff G, Carnevale P, Fondjo E, Robert V: Comparison of three sampling methods of man-biting anophelines in order to estimate the malaria transmission in a village of south Cameroon. Parasite 1997, 4:75-80.

33. Awolola TS, Okwa O, Hunt RH, Ogunrinade AF, Coetzee M: Dynamics of the malaria-vector populations in coastal Lagos, south-western Nigeria. Ann Trop Med Parasitol 2002, 96:75-82.

34. Fontenille D, Lochouarn L, Diatta M, Sokhna C, Dia I, Diagne N, Lemasson JJ, Ba K, Tall A, Rogier C, Trape JF: Four years' entomological study of the transmission of seasonal malaria in Senegal and the bionomics of Anopheles gambiae and A. arabiensis. Trans $R$ Soc Trop Med Hyg 1997, 91:647-652.

35. Smith DL, Guerra CA, Snow RW, Hay SI: Standardizing estimates of the Plasmodium falciparum parasite rate. Malar J 2007, 6:131.

36. Hay SI, Noor AM, Simba M, Busolo M, Guyatt HL, Ochola SA, Snow RW: Clinical epidemiology of malaria in the highlands of Western Kenya. Emerg Infect Dis 2002, 8:543-548.

37. Hay SI, Rogers DJ, Toomer JF, Snow RW: Annual Plasmodium falciparum entomological inoculation rates (EIR) across Africa: literature survey, internet access and review. Trans R Soc Trop Med Hyg 2000, 94:113-127.

38. Chalvet-Monfray $K$, Sabatier P, Bicout DJ: Downscaling modeling of the aggressiveness of mosquitoes vectors of diseases. Ecol Model 2007, 204:540-546.

39. Shaman J, Day JF: Achieving operational hydrologic monitoring of mosquitoborne disease. Emerg Infect Dis 2005, 11:1343-1350.

40. Balme M, Vischel T, Lebel T, Peugeot C, Galle S: Assessing the water balance in the Sahel: impact of small scale rainfall variability on runoff. Part 1: Rainfall variability analysis. Journal of Hydrology 2006, 331:336-348.

41. Murphy AH: Skill scores based on the mean square error and their relationships to the correlation coefficient. Monthly Weather Review 1988, 25:2417-2424.

42. Ahumada JA, Lapointe D, Samuel MD: Modeling the population dynamics of Culex quinquefasciatus (Diptera: Culicidae), along an Elevational Gradient in Hawaii. J Med Entomol 2004, 41:1157-70.

43. MARA: Towards an atlas of malaria risk in Africa: first technical report of the MARA/ARMA collaboration. First technical report, MARA/ARMA Durban, South Africa; 1998.

44. Le Masson JJ, Fontenille D, Lochouam L, Dia I, Simard F, Ba K, Diop A, Diatta M, Molez JF: Comparison of behavior and vector efficiency of Anopheles gambiae and An. arabiensis (Diptera: Culicidae) in Barkedji, a Sahelian area of Senegal. J Med Entomol 1997, 34:396-403.

45. Molez JF, Diop A, Gaye O, Lemasson JJ, Fontenille D: Malaria morbidity in Barkedji, village of Ferlo, in Senegal Sahelian area. Bull Soc Pathol Exot 2006, 99:187-190.

46. Stafford Smith DM: Mosquito records from the Republic of Niger, with reference to the construction of the new 'Trans-Sahara Highway'. J Trop Med Hyg 1981, 84:95-100.

47. Snow RW, Craig M, Deichmann U, Marsh K: Estimating mortality, morbidity and disability due to malaria among Africa's non-pregnant population. Bull World Health Org 1999, 77:624-640.

48. Rogers DJ, Randolph SE, Snow RW, Hay SI: Satellite imagery in the study and forecast of malaria. Nature 2002, 415:710-715. 
49. Gemperli A, Sogoba N, Fondjo E, Mabaso M, Bagayoko M, Brit OJT, Anderegg D, Liebe J, Smith T, Vounatsou P: Mapping malaria transmission inWest and Central Africa. Trop Med Int Health 2006, 11:1032-1046.

50. Shaman J, Day JF: Reproductive phase locking of mosquito populations in response to rainfall frequency. PLoS One 2007, 3:e331.

51. Guerra CA, Hay SI, Lucioparades LS, Gikandi PW, Tatem AJ, Noor AM, Snow RW: Assembling a global database of malaria parasite prevalence for the Malaria Atlas Project. Malar J 2007, 6:17.

52. Kleinschmidt I, Omumbo J, Briët O, van de Giesen N, Sogoba N, Mensah NK, Windmeijer P, Moussa M, Teuscher T: An empirical malaria distribution map for West Africa. Trop Med Int Health 2001, 6:779-786.

53. Epstein PR: Global warming and vector-borne disease. Lancet 1998, 351:1737.

54. van Lieshout M, Kovats RS, Livermore MTJ, Martens P: Climate change and malaria: analysis of the SRES climate and socio-economic scenarios. Glob Environ Change 2004, 14:87-99.

55. Briët OJT: A simple method for calculating mosquito mortality rates, correcting for seasonal variations in recruitment. Med Vet Entomol 2002, $16: 22-27$

56. Munga S, Minakawa N, Zhou G, Mushinzimana E, Barrack OOJ, Githeko AK, Yan G: Association between land cover and habitat productivity of malaria vectors in western Kenyan highlands. Am J Trop Med Hyg 2006, 74:69-75.

57. Keiser J, Utzinger J, de Castro MC, Smith TA, Tanner M, Singer BH: Urbanization in sub-Saharan Africa and malaria control. Am J Trop Med Hyg 2004, 71(Suppl. 2):118-127.

doi:10.1186/1475-2875-10-62

Cite this article as: Ermert et al: Development of a new version of the Liverpool Malaria Model. II. Calibration and validation for West Africa. Malaria Journal 2011 10:62.

\section{Submit your next manuscript to BioMed Central and take full advantage of:}

- Convenient online submission

- Thorough peer review

- No space constraints or color figure charges

- Immediate publication on acceptance

- Inclusion in PubMed, CAS, Scopus and Google Scholar

- Research which is freely available for redistribution

Submit your manuscript at www.biomedcentral.com/submit 